\title{
Synthesis and catalytic application of ferrocene substituted camphane-based aminoalcohols and $S$-containing heterocyclic analogues
}

\author{
Mariana Kamenova-Nacheva, Georgi M. Dobrikov and Vladimir Dimitrov* \\ * Institute of Organic Chemistry with Center of Phytochemistry, Bulgarian Academy of Science, Acad. G. Bonchev 9, Sofia 1113, Bulgaria
}

\begin{abstract}
A facile and practical approach was developed for the synthesis of chiral $\alpha$-, $\beta$ - and $\delta$-aminoalcohols and $S$-containing heterocyclic analogues by using of readily available sources of chirality. The synthetic strategy involves utilization of camphorderived ferrocene-containing starting compounds allowing, by means of nucleophilic additions of functionalized organolithiums, for the preparation of a small library of structurally diverse multifunctional derivatives possessing the bicyclic camphane skeleton as the central core. The new diastereoisomeric pure aminoalcohols and $S$-containing heterocyclic analogues have been evaluated as precatalysts for the addition of diethyl zinc to benzaldehyde providing in some cases very high enantioselectivity (up to $96 \%$ ).
\end{abstract}

\section{Introduction}

The enantioselective addition of organozinc reagents to carbonyl compounds observed by Oguni and Omi, ${ }^{1}$ first demonstrated with the reaction of $\mathrm{Et}_{2} \mathrm{Zn}$ and benzaldehyde catalyzed by $(S)$-leucinol, still attracts considerable interest due to the opportunity of generating important pharmaceutically relevant intermediates with high enantioselectivity. ${ }^{2-5}$ The methods developed in recent years for the synthesis of structurally diverse organozinc reagents $^{6-8}$ and a wide variety of chiral aminoalcohols ${ }^{9,10}$ prepared with the purpose to catalyze enantioselectively the additions of organozinc reagents, led the motivation to design the synthesis of new important chiral compounds. Since Noyori et al. ${ }^{11-16}$ showed the efficiency of 3-dimethylamino-isoborneol as a chiral aminoalcohol able to serve as a ligand, there has been considerable interest for the preparation of structurally diverse aminoalcohols incorporating the camphane skeleton.

For the synthesis of camphene-based derivatives containing different functionalities camphor and fenchone are very convenient chiral pool sources which are readily available in enantiomerically pure form. The realization of diverse substitutions at the 1-, 2-, 3- and 7-positions of the camphane skeleton has been achieved by introducing mainly hydroxy and/or heteroatom-containing groups (Fig.1; the methyl groups in the product representing the different substitutions are omitted for clarity). Several synthetic strategies have been developed for the introduction of functionalities at 1- and 2position of the bicyclic skeleton, ${ }^{17-32}$ some of which include skeleton rearrangements. ${ }^{33-37}$ The most simple way to introduce simultaneously hydroxyl- and heteroatom-containing groups at the 2-position is the nucleophilic addition of functionalized organometallic reagents to camphor and fenchone leading directly ${ }^{38-47}$ or after subsequent transformations $^{48-51}$ to aminoalcohols or analogues. The simultaneous occupation of the 2- and 3-position is limited to the synthesis of 3-dimethylaminoisoborneol and analogues. ${ }^{52,53}$ Interesting approaches have been demonstrated to introduce functionalities at the 2- and 7-position of the camphane scaffold ${ }^{54,55}$ and to realize the rare example of 1-, 2and 3 -substitution. ${ }^{56}$

\section{sources of chirality}

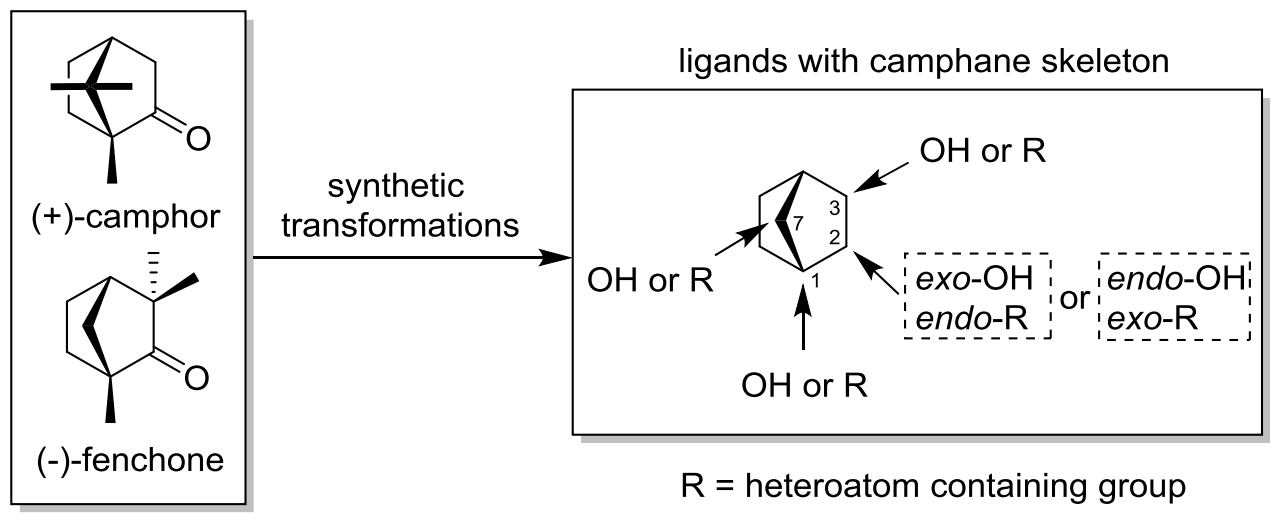

Figure 1. Substitution pattern within the camphane skeleton realized by synthetic transformations from camphor and fenchone (regardless the positions of remaining methyl groups).

In recent years we have demonstrated the synthesis of $2,3-{ }^{57}$ and $1,2,3$-substituted ${ }^{58}$ camphane-based aminoalcohols and analogues. The ability of these derivatives to act as precatalysts for enantioselective additions of 
$\mathrm{Et}_{2} \mathrm{Zn}$ to aldehydes has been evaluated as promising. Herein we report a synthetic approach to obtain aminoalcohols and analogues starting from (+)-camphor $\mathbf{1}$ and (+)-camphor-10-sulfonamide $\mathbf{3}$ by shielding the C-3 position with ferrocenecontaining a fragment and then by using the nucleophilic addition approach to realize C-2 substitution.

\section{Results and discussion}

The synthesis of the starting compounds $\mathbf{7}$ and $\mathbf{9}$ for the planned addition reactions was performed by the initial generation of chiral enolates $\mathbf{2}$ and $\mathbf{4}$ (Scheme 1) from (+)-camphor $\mathbf{1}$ and (+)-10-camphor-sulfonamide ,3 and subsequent Claisen-Schmidt condensation with ferrocene carbaldehyde. The enolates generation ( 2 and $\mathbf{4})$ was realized by deprotonation of $\mathbf{1}$ and $\mathbf{3}$ with lithium bis(trimethylsilyl)amide (LiHMDS) ${ }^{58}$ or LDA (Method A), or alternatively by reaction with $\mathrm{KOH}$ under phase-transfer conditions catalyzed by 18-crown-6 (Method B) following an analogously published procedure. ${ }^{59}$ The condensation using Method A is faster, however the yields of 7 and 9 do not exceed $70 \%{ }^{58}$ Method B provides 7 and 9 in excellent yields (up to 91\%) but requires longer reaction times. In all cases, the $Z$-isomers $\mathbf{6}$ and $\mathbf{8}$ are formed (up to 6\%), which can be easily separated by column chromatography. Method B is suitable to perform the synthesis of $\mathbf{7}$ and $\mathbf{9}$ on a multi-gram scale.
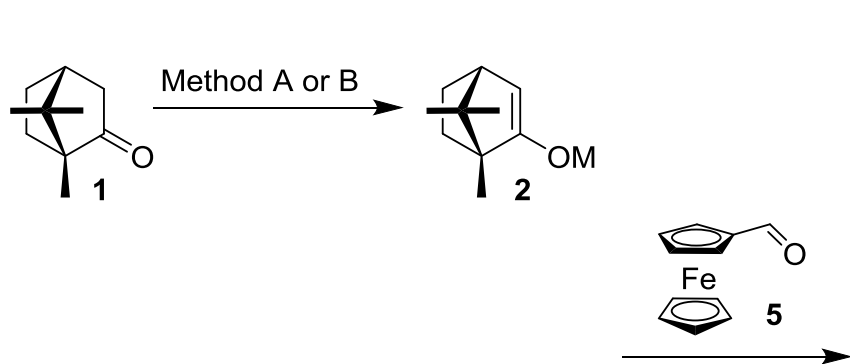

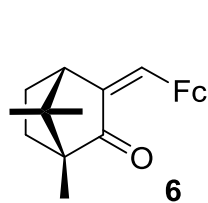

(Z-configuration) A: $6 \%$ B: $4 \%$

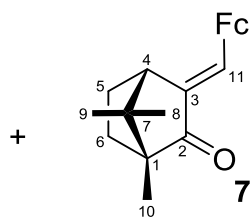

(E-configuration) $60 \%$ $88 \%$
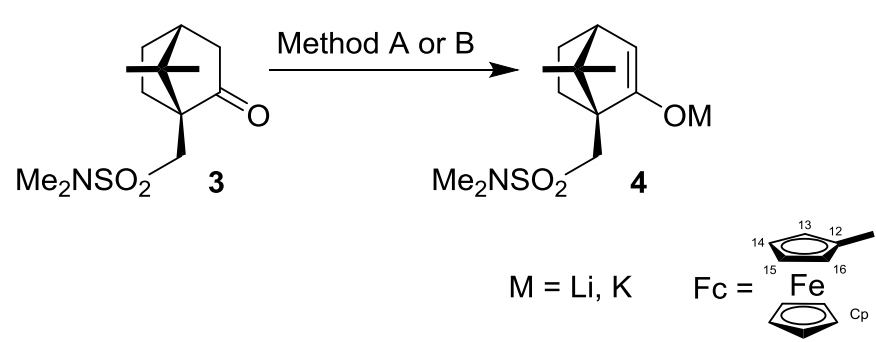<smiles>COS(C)(=O)=O</smiles>

(Z-configuration) A: $5 \%$

B: $2 \%$

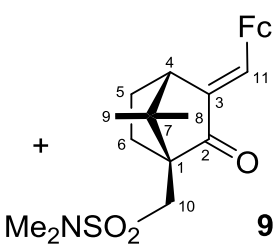

(E-configuration) $59 \%$ $91 \%$

Scheme 1. Synthesis of the starting compounds 7 and 9.

Method A: LiHMDS or LDA for 2 and 4, respectively; Method B: KOH/18-crown-6.

The selection of the functionalized organolithium reagents for the additions to $\mathbf{7}$ and $\mathbf{9}$ was performed in accordance with previously published results. ${ }^{39,42,44,60}$ Reagents $\mathbf{1 0 - 1 2}{ }^{61,62}$ and $\mathbf{1 4}^{62}$ were generated in situ and used in hexane, hexane/ether or THF solvent mixtures (Scheme 2). Reagent 13 was isolated ${ }^{63}$ and applied in pure form (colorless crystals). The addition of reagents 10-14 to ketone 7 provided products 15-19 in low to moderate yields (Scheme 2). The reaction of reagent 10 with 7 produced compound 15 in $17 \%$ yield together with a significant amount of 1,4-addition product 20 (20\%). The addition of 11 consumed 7 completely, however significant decomposition occurred (recognized by TLC through the presence of several not identified by-products) thus lowering the yield of $\mathbf{1 6}$ (19\% yield). With the analogous reagent 12, the formation of product $\mathbf{1 7}$ was significantly higher (33\%). The addition of reagents 13 and 14 in hexane was very low yielding (5\% of 18 and $8 \%$ of 19) as a result of a considerable 1,4-addition reaction ( $40 \%$ of $\mathbf{2 1}$ and $28 \%$ of $\mathbf{2 2}$ were isolated). In the case of products $\mathbf{1 5}$ and $\mathbf{1 7}$, considerable amounts of the starting ketone 7 were isolated (37\% and 53\%, respectively) as result of its low conversion. The activation of 7 with anhydrous $\mathrm{CeCl}_{3}$ in $\mathrm{THF}^{64}$ improved only the addition of reagents $\mathbf{1 3}$ and $\mathbf{1 4}$ resulting in the isolation of the desired products 18 and 19 in higher yields (53\% and 50\%, respectively). Despite the $\mathrm{CeCl}_{3}$ activation of ketone 7, significant amounts of 1,4-addition product $\mathbf{2 1}$ were isolated after the reaction of reagent $\mathbf{1 3}$. The competing 1,4-additon reaction is the main reason for the low yields of the desired products. 


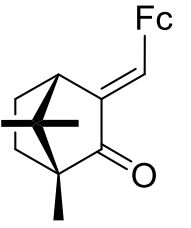

7

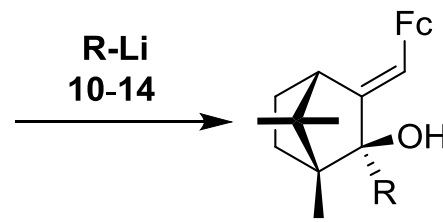

15-19

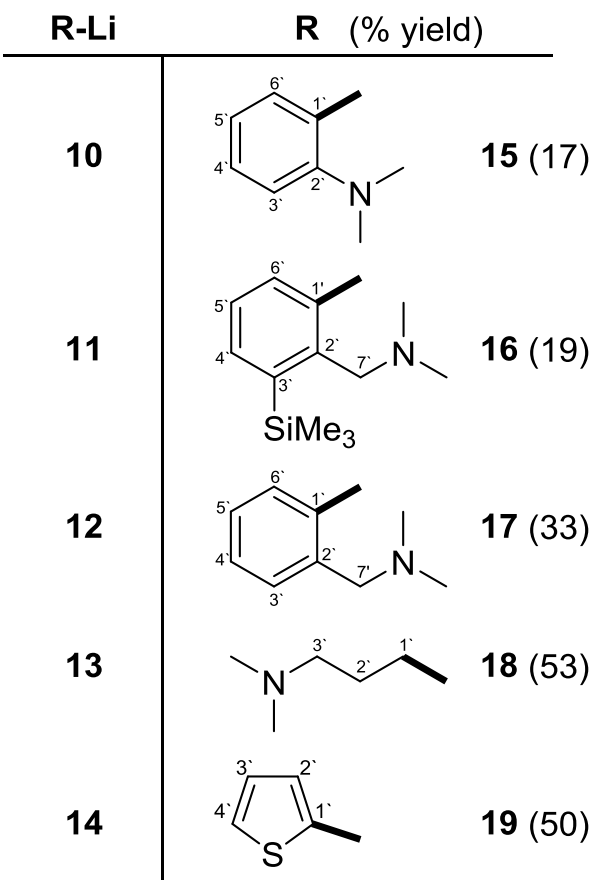

Isolated products of 1,4-addition:

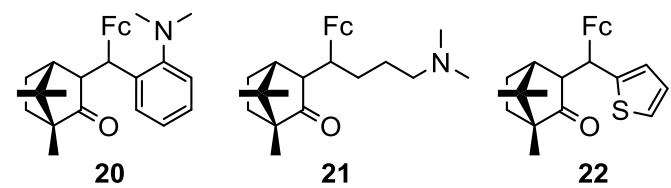

Scheme 2. Synthesis of the compounds $\mathbf{1 5 - 1 9}$ by addition of organolithium reagents 10-14 to 7 .

The direct synthesis of ferrocene-substituted aminoalcohols and sulfur-containing analogues by using the addition approach of organolithium reagents was also applied in the case of ketone 9 (Scheme 3). The addition of reagents 10-12 to the ferrocenylmethylidene-camphor-10-sulfonamide $\mathbf{9}$ was not practical; reagent $\mathbf{1 0}$ did not react with $\mathbf{9}$ and the addition of $\mathbf{1 2}$ provided only the 1,4-addition product (mixture of two diastereoisomers in $65 \%$ yield). The latter observation was the reason for not performing reactions with reagent $\mathbf{1 1}$ since a similar result was expected. The addition of reagents $\mathbf{1 3}$ and $\mathbf{2 3 - 2 8}{ }^{62,65-68}$ (generated in situ) to ketone $\mathbf{9}$ was performed in THF or hexane/THF solvent mixtures (in case of $\mathbf{1 3}$ toluene was used). The yields of the isolated 1,2-addition products were moderate (up to $48 \%$ ). In the case of reagents $\mathbf{1 3}, \mathbf{2 3}$ and $\mathbf{2 6}$, considerable amounts of 1,4-additon products 36-38 were isolated in form of inseparable mixtures of diastereoisomers. The reaction of reagents $\mathbf{2 4}, \mathbf{2 7}$ and $\mathbf{2 8}$ with 9 provided moderate yields of products $\mathbf{3 1}, \mathbf{3 4}$ and $\mathbf{3 5}$ (in 48\%, 47\% and $25 \%$ yields of isolated pure compounds, respectively). Significant amounts of unreacted ketone 9 could be observed in most cases. The difficult separation of the products from mixtures containing 9 was an additional reason for reduced yields.

Some aspects of the 1,4-addition are ntoeoworthy. In most cases, we observed the formation of two diastereoisomers. Compound $\mathbf{2 0}$ was isolated as a single diastereoisomer while in the case of 21 (procedure A; see Experimental) three isomers were observed in the ratio 5:10:12 contrary to procedure $\mathrm{B}$, which provided only two isomers (ratio 2:5). In general, the formation of the 1,4-addition products depended strongly on the lithium reagent applied. The use of anhydrous $\mathrm{CeCl}_{3}$ could shift some of the reactions towards the 1,2-addition, but not completely. The isolation of pure isomers was possible for $\mathbf{3 7}$ and $\mathbf{3 8}$ by column chromatography after significant efforts. The 
experimental data with respect to the isolation, purification and determination of the ratio observed are reported in the Experimental. It was not possible to collect enough data for assigning the isomers either as originating from endo- or exo-positioned side chain, and/or as a result of the stereogenic center formation next to the ferrocene moiety.
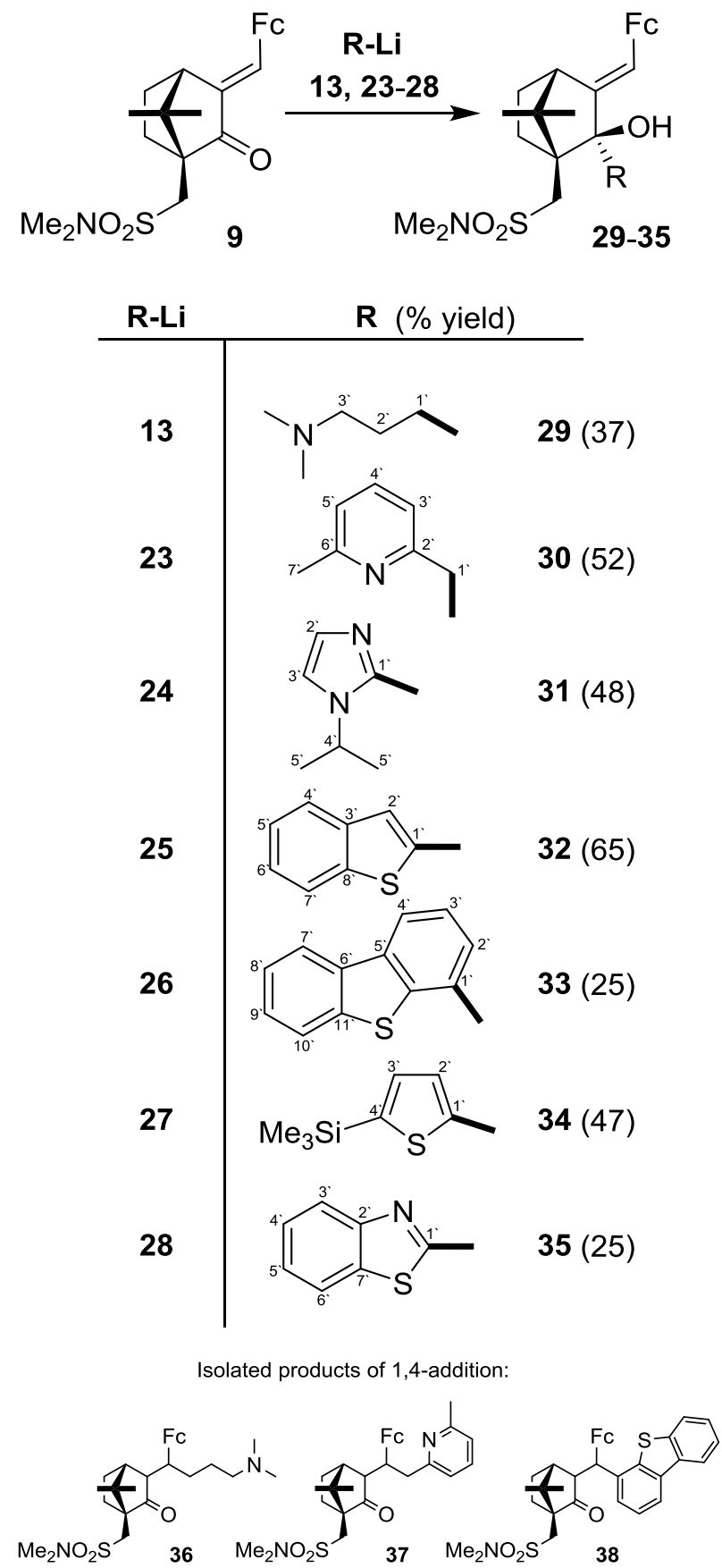

Scheme 3. Synthesis of compounds 29-30 by the addition of organolithium reagents $\mathbf{1 3}$ and 23-28 to 9.

A further reaction sequence leading to aminoalcohols was the addition of reagent $39\left(\mathrm{LiCH}_{2} \mathrm{CN}\right.$ generated in situ from $n$-BuLi and dry $\mathrm{CH}_{3} \mathrm{CN}$ ) ${ }^{69,70}$ to ketones 7 and 9 (Scheme 4). The addition was performed at $-78^{\circ} \mathrm{C}$ in $\mathrm{THF}$ to give compounds $\mathbf{4 0}$ and 41, respectively, in good yields, which were then reduced to the corresponding aminoalcohols 42 and 43. The latter were transformed easily into the alkylated cyclic derivatives 45 and 46 by using a simple procedure (addition of compound $\mathbf{4 4}$ and excess of $\mathrm{K}_{2} \mathrm{CO}_{3}$ in refluxing acetonitrile). ${ }^{71}$ 


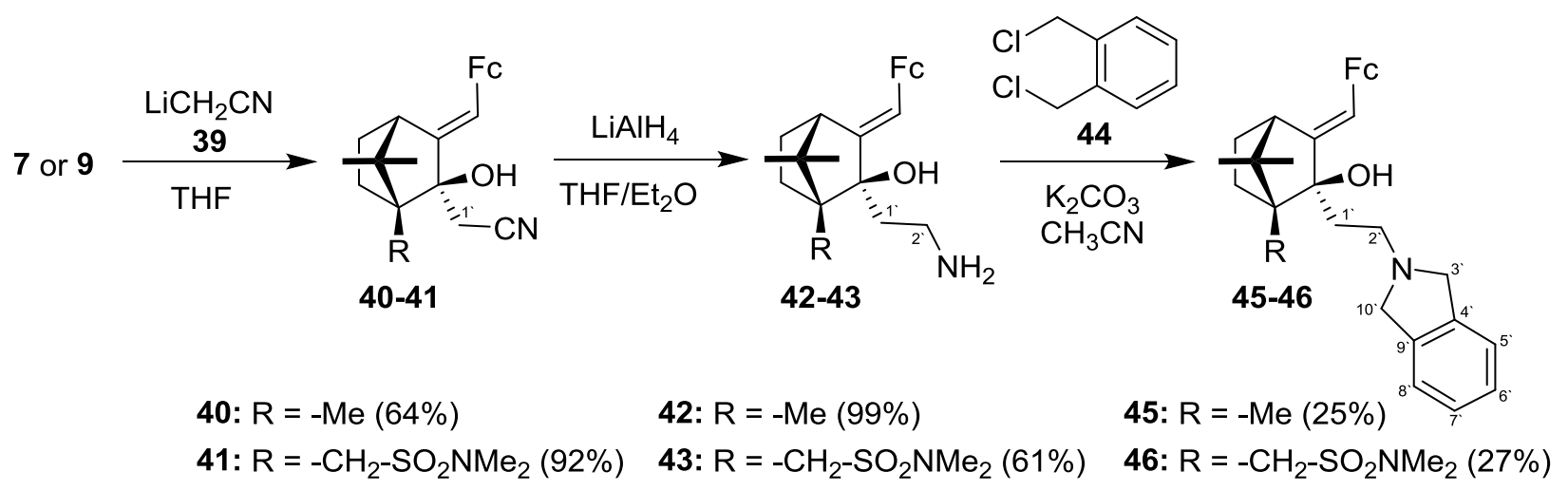

Scheme 4. Synthesis of compounds 45 and $\mathbf{4 6}$ by the addition of cyanomethyl lithium 39 to 7 and 9 and subsequent transformations.

The structures of the new chiral compounds were proved by NMR experiments and mass spectra. The unambiguous assignment of the ${ }^{1} \mathrm{H}$ and ${ }^{13} \mathrm{C}$ NMR spectra was made on the basis of DEPT, COSY, HSQC, HMBC and NOESY experiments. In all cases, the observed endo-diastereoselectivities of the addition reactions to the carbonyl Catom of ketones $\mathbf{7}$ and $\mathbf{9}$ were excellent due to the exclusive endo-attack of the organolithium reagents. The decisive arguments for the course of the additions and thus for the determination of configurations were the NOESY experiments.

For compounds 15-17 and 19, characteristic NOESY signals confirmed the proximity between ortho-aromatic protons of the substituents and $5-\mathrm{H}_{\text {endo }} / 6-\mathrm{H}_{\text {endo }}$ protons were observed (Fig. 2). In the case of 45 strong interaction between both protons of the $\mathrm{CH}_{2}$-group and 5- $\mathrm{H}_{\text {endo }} / 6-\mathrm{H}_{\text {endo }}$ protons was observed. Compound 18 was isolated as a single diastereoisomer, but due to signals overlap for $\mathrm{CH}_{2}$-protons (from the side chain) and 5- $\mathrm{H}_{\text {endo }} / 6-\mathrm{H}_{\text {endo }}$ protons, no essential interactions could be observed. However, according to previously published results ${ }^{44}$ for similar camphorderived compounds, an exo-position for the $\mathrm{OH}$-group in $\mathbf{1 8}$ was presumed. The observed proximity between $\mathrm{OH}$ protons and 10-H/11-H in 15-17 and 19 confirmed the exo-position of the OH-groups in these compounds.

To confirm the endo-position of the substituents in compounds 30-34 and 46, the same approach was applied (Fig. 2). In all cases characteristic protons from the substituents were determined to have close proximities to at least one of the endo-protons of the bicyclic moiety. In the case of compound $\mathbf{3 5}$ there is no suitable proton within the introduced substituent to demonstrate characteristic proximity to endo-positioned protons. However, there is an interaction observed in the NOESY spectra, between the protons of the OH-group and the protons of the methyl group (C-8) attached to the C-bridge of the bicyclic moiety. The same proton proximities are observed in the case of compounds 32-34. For compound 29, no characteristic signals could be observed due to overlapping resonances of the $\mathrm{CH}_{2}$-protons from the substituent chain and the $5-\mathrm{H}_{\text {endo }} / 6-\mathrm{H}_{\text {endo }}$ protons of the bicyclic core. The endo-position of the dimethylaminopropyl group could also be deduced in this case from previous results. ${ }^{44}$ 

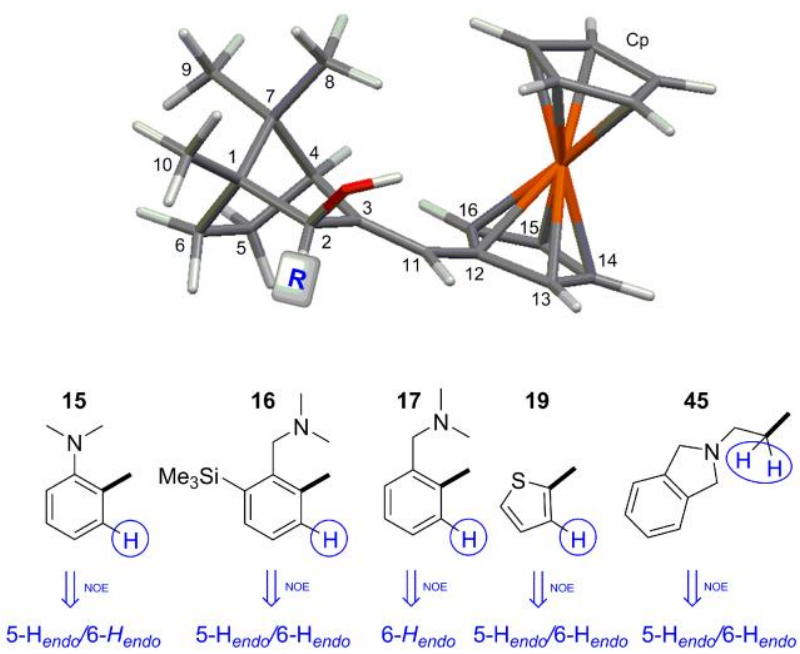
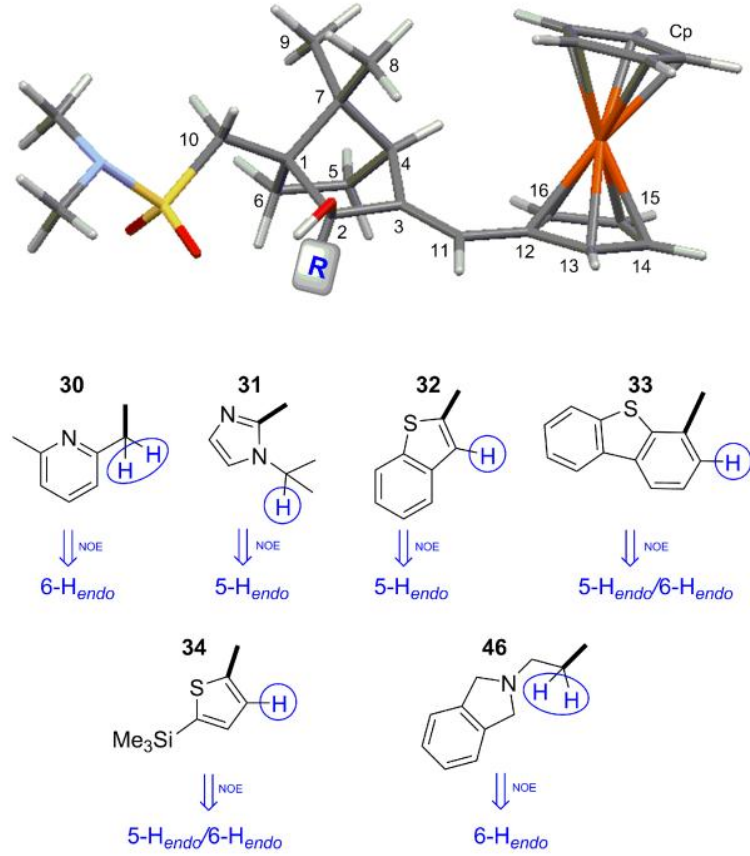

Figure 2. The observed proton proximities of specified substituent protons and the endo-H within the bicyclic core of compounds 15-17, 19, 30-34, 45 and 46, obtained by means of NOESY spectra.

Compounds 15-19, 29-35, 45 and 46 were applied as ligands for the enantioselective addition of $\mathrm{Et}_{2} \mathrm{Zn}_{\mathrm{n}}$ to benzaldehyde (Table 1) according to the literature. ${ }^{58}$ The reaction times were between 3 and 168 hours. The yields of the isolated 1-phenyl-1-propanol were excellent in all cases, except for the reaction catalyzed by ligand $\mathbf{3 0}$ (entry 7, Tab. 1). The enantioselectivities obtained were in the range of low to excellent (up to 96\%). The best enantioselectivities were realized with ligands $\mathbf{1 5}$ and 16. When comparing ligands $\mathbf{1 6}$ and $\mathbf{1 7}$ there is a clear influence of the $\mathrm{Me}_{3} \mathrm{Si}$-group for obtaining better enantioselectivity. The same trend has been reported. ${ }^{42}$ The moderate enantioselectivity obtained with ligands 18 and $\mathbf{2 9}$ is in the range of those observed for the analogous ligand prepared through the reaction of reagent $\mathbf{1 3}$ and (+)-camphor. ${ }^{44}$ It is interesting to note that ligands $\mathbf{4 7}$ and $\mathbf{4 8}$, whose synthesis and application has been published earlier, ${ }^{58}$ provide better enantioselectivities than ligands $\mathbf{1 9}$ and 32. The latter are sulfur-containing structural analogues of $\mathbf{4 7}$ and $\mathbf{4 8}$. By comparing the thiophene-substituted ligands 19 and $49,{ }^{58}$ it seems that there is a positive influence of the sulfonamide group leading to an improvement of the enantioselectivity.

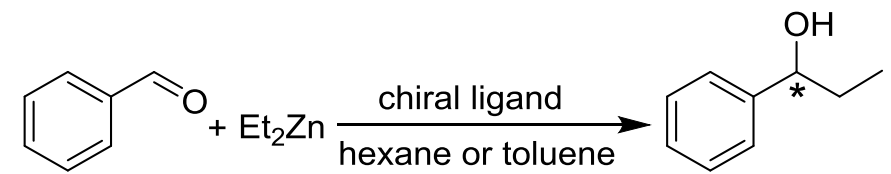

Scheme 5. Addition of $\mathrm{Et}_{2} \mathrm{Zn}$ to benzaldehyde catalyzed by chiral ligands 15-19, 29-35 and 45-49.

Table 1. Addition of $\mathrm{Et}_{2} \mathrm{Zn}$ to benzaldehyde catalyzed by ligands 15-19, 29-35 and 45-49.

\begin{tabular}{ccccr} 
Entry & Ligand & $\begin{array}{c}\text { Ligand } \\
(\% \text { mol })\end{array}$ & $\begin{array}{c}\text { Yield } \\
(\%)^{\mathrm{a}}\end{array}$ & $\begin{array}{r}\text { ee }(\%), \\
\text { conf }^{\mathrm{b}}\end{array}$ \\
\hline 1 & $\mathbf{1 5}$ & 1.5 & 99 & $96(R)$ \\
2 & $\mathbf{1 6}$ & 1.5 & 97 & $96(S)$ \\
3 & $\mathbf{1 7}$ & 3.0 & 99 & $89(S)$ \\
4 & $\mathbf{1 8}$ & 1.5 & 96 & $35(R)$ \\
5 & $\mathbf{1 9}$ & 3.0 & 96 & $22(R)$ \\
6 & $\mathbf{2 9}$ & 3.0 & 82 & $68(S)$ \\
7 & $\mathbf{3 0}$ & 3.0 & 62 & $14(R)$ \\
8 & $\mathbf{3 1}$ & 3.0 & 97 & $20(S)$ \\
9 & $\mathbf{3 2}$ & 3.0 & 90 & $62(R)$ \\
10 & $\mathbf{3 3}$ & 3.0 & 73 & $6(S)$ \\
11 & $\mathbf{3 4}$ & 3.0 & 92 & $56(R)$ \\
12 & $\mathbf{3 5}$ & 3.0 & 90 & $18(S)$ \\
13 & $\mathbf{4 5}$ & 3.0 & 99 & $38(R)$
\end{tabular}




\begin{tabular}{|c|c|c|c|c|}
\hline 14 & 46 & 3.0 & 88 & $25(R)$ \\
\hline 15 & $47^{\mathrm{c}}$ & 3.0 & 59 & $76(R)$ \\
\hline 16 & $48^{\mathrm{c}}$ & 3.0 & 83 & $76(\mathrm{R})$ \\
\hline 17 & $49^{c}$ & 3.0 & 48 & $56(\mathrm{R})$ \\
\hline
\end{tabular}

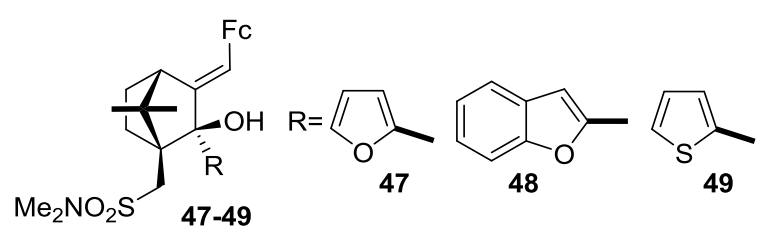

\section{Conclusion}

In conclusion, we have demonstrated a practicable application of functionalized organolithium reagents for the synthesis of chiral $\beta$-, $\gamma$ - and $\delta$ - aminoalcohols as well as $S$-containing analogues possessing the bicyclo-heptane skeleton as the central core and ferrocene moiety as the bulky substituent attached to the 3-position of the bicyclic core. The use of inexpensive and readily available sources of chirality makes the presented synthetic strategies very useful for the preparation of a structurally diverse series of multifunctional chiral derivatives. The series of compounds synthesized were isolated in diastereoisomerically pure form and were characterized by spectroscopic methods. The configurations, with respect of the newly formed stereogenic centers within the compounds synthesized, were determined by applying advanced NMR methods. The evaluation of the synthesized compounds as ligands in the addition of $\mathrm{Et}_{2} \mathrm{Zn}$ to benzaldehyde provided moderate to excellent enantioselectivities (up to $96 \%$ ). The synthesized small library of compounds was evaluated for the biological activity and showed promising results (these results will be published elsewhere).

\section{Experimental}

\subsection{General}

The reactions were carried out in flame-dried Schlenk flasks under an argon atmosphere. Tetrahydrofuran (THF) and diethyl ether $\left(\mathrm{Et}_{2} \mathrm{O}\right)$ were distilled over sodium/benzophenone. Hexane, benzene and toluene were distilled over $\mathrm{Na}\left[\mathrm{Et}_{4} \mathrm{Al}\right]$. Acetonitrile was dried over molecular sieve ( $3 \AA$ ). Thin layer chromatography (TLC): aluminum sheets pre-coated with silica gel $60 \mathrm{~F}_{254}$ (Merck). Column chromatography was carried out at normal pressure, using silica gel 60 (0.040-0.063 mm, 230-400 mesh ASTM Merck). Methyl tert-butyl ether (MTBE) and other solvents for crystallization, TLC and column chromatography were of high purity (>99.8\%) and were used without distillation. Melting points were determined in capillary tubes on Electrothermal MEL-TEMP 1102D-230 VAC apparatus without corrections. Optical rotation $[\alpha]$ measurements were obtained using Perkin-Elmer 241 polarimeter. Enantiomeric excess was measured on gas chromatograph Shimadzu GC-17A equipped with flame ionization detector (FID) and two chiral capillary columns: Cyclosil-B (permethylated- $\beta$-cyclodextrin) $(30 \mathrm{~m}, \varnothing=0.25 \mathrm{~mm}$, film thickness $0.25 \mu \mathrm{m}$; eedetermination of 1-phenyl-1-propanol: $0.7 \mathrm{ml} / \mathrm{min} \mathrm{He}$ flow; thermal gradient $10 \mathrm{~min}$ at $80^{\circ} \mathrm{C}, 2^{\circ} \mathrm{C} / \mathrm{min}$ to $100^{\circ} \mathrm{C}, 10 \mathrm{~min}$ at $100,3^{\circ} \mathrm{C} / \mathrm{min}$ to $150^{\circ} \mathrm{C}$; retention time $\mathrm{t}_{(R)}=41.4 \mathrm{~min}, \mathrm{t}_{(S)}=42.2 \mathrm{~min}$ ) and Hydrodex- $\beta$-TBDAc (heptakis- $(2,3$-di-Oacetyl-6-O-t-butyldimethyl-silyl)- $\beta$-cyclodextrin) $(25 \mathrm{~m}, \varnothing=0.25 \mathrm{~mm}$; ee-determination of 1-phenyl-1-propanol: 0.7 $\mathrm{ml} / \mathrm{min}$ He flow; isothermal $122^{\circ} \mathrm{C}$; retention time $\left.\mathrm{t}_{(R)}=9.4 \mathrm{~min}, \mathrm{t}_{(S)}=9.8 \mathrm{~min}\right)$. Mass spectra $(\mathrm{MS})$ were recorded on a Thermo Scientific HRDFS (High Resolution Double Focusing Magnetic Sector) mass spectrometer (Bremen, Germany) and on a Hewlett Packard Mass Selective Detector 5973. MS spectra were reported as fragmentation in $\mathrm{m} / \mathrm{z}$ with relative intensities (\%) in parentheses. NMR spectra were recorded on a Bruker Avance DRX-250 $\left({ }^{1} \mathrm{H}\right.$ at $250.13 \mathrm{MHz} ;{ }^{13} \mathrm{C}$ at $62.90 \mathrm{MHz})$ and Bruker Avance II+ $600\left({ }^{1} \mathrm{H}\right.$ at $600.13 \mathrm{MHz} ;{ }^{13} \mathrm{C}$ at $\left.150.92 \mathrm{MHz}\right)$ spectrometers with TMS as internal standard for chemical shifts $(\delta, \mathrm{ppm})$. For the numbering of the $\mathrm{C}$-atoms see Schemes $1-4 .{ }^{1} \mathrm{H}$ and ${ }^{13} \mathrm{C}$ NMR data are reported as follows: chemical shift, multiplicity $(\mathrm{s}=$ singlet, $\mathrm{d}=$ doublet, $\mathrm{t}=$ triplet, $\mathrm{q}=$ quartet, $\mathrm{m}=$ multiplet; br $=$ broad signal), integration, identification and coupling constants $(\mathrm{Hz})$. The chemical shifts of the ambiguously assigned signals in NMR data are marked with an asterisk (Cp stands for cyclopentadienyl, Fc marks the ferrocene moiety). The assignment of the ${ }^{1} \mathrm{H}$ and ${ }^{13} \mathrm{C}$ NMR spectra was made on the basis of DEPT, COSY, HSQC, HMBC and NOESY experiments. Samples for NOE difference experiments were prepared by blowing argon through the $\mathrm{CDCl}_{3}$ solution. Elemental analyses were performed by the Microanalytical Laboratory for Elemental Analysis at the Institute of Organic Chemistry, Bulgarian Academy of Sciences. The following starting materials were used (commercially available or prepared according to the literature): ferrocenecarbaldehyde (Acros), $n$-butyllithium ( $n$-BuLi, Fluka), (+)-camphor 
(Fluka), diethylzinc (1 M solution in hexane, Fluka), N,N-dimethyl(2-(trimethylsilyl)phenyl)methanamine, ${ }^{42}$ (3(dimethylamino)propyl)lithium, ${ }^{63}$ thiophene (Fluka), $N, N$-dimethylbenzylamine (Fluka), $N, N$-dimethylaniline (Fluka), lithium aluminium hydride (Fluka), lithium diisopropylamide (LDA, Fluka), lithium hexamethyldisilazide (LiHMDS, Fluka), tetramethylethylenediamine (TMED), anhydrous cerium (III) chloride, ${ }^{46} 1$-isopropyl- $1 H$-imidazole, ${ }^{68} 2,6$ lutidine (Fluka), benzothiophene (Fluka), dibenzothiophene (Fluka), trimethy-2-thienylsilane (Acros), benzothiazole (Fluka), 18-crown-6 (Fluka), $\alpha, \alpha^{\prime}$-dichloro-o-xylene (Aldrich).

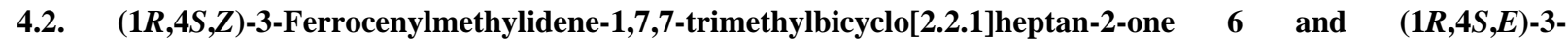
ferrocenylmethylidene-1,7,7-trimethylbicyclo[2.2.1]heptan-2-one 7

Method A:

To a solution of $1(2.30 \mathrm{~g}, 15.00 \mathrm{mmol})$ in $100 \mathrm{ml}$ of dry toluene was added LDA $(7.50 \mathrm{ml}, 15.12 \mathrm{mmol}$ of $2 \mathrm{M}$ solution in THF/ethylbenzene). After stirring for $15 \mathrm{~min}, 5(2.10 \mathrm{~g}, 10.00 \mathrm{mmol})$ was added and the deep red mixture refluxed for $1 \mathrm{~h}$. The reaction was monitored by TLC (petroleum ether/ $\mathrm{Et}_{2} \mathrm{O}=5: 1$ ). The mixture was cooled and quenched with saturated aq. $\mathrm{NH}_{4} \mathrm{Cl}$, extracted with $\mathrm{Et}_{2} \mathrm{O}$ and the organic phase was dried over anhydrous $\mathrm{Na}_{2} \mathrm{SO}_{4}$. The solvent was evaporated in vacuo and the crude product was chromatographed $\left(37 \mathrm{~g}\right.$ silica gel, petroleum ether/Et ${ }_{2} \mathrm{O}=$ $25: 1)$ to give $6(0.170 \mathrm{~g}, 6 \%)$ as dark red crystals and $7(2.05 \mathrm{~g}, 60 \%)$ as deep red crystals.

Method B:

A mixture of $1(0.50 \mathrm{~g}, 3.28 \mathrm{mmol}), \mathrm{KOH}(0.28 \mathrm{~g}, 4.92 \mathrm{mmol})$ and 18 -crown-6 (0.087 g, $0.33 \mathrm{mmol}) \mathrm{in} 10 \mathrm{ml}$ dry benzene was stirred under argon for $30 \mathrm{~min}$ and $5(0.70 \mathrm{~g}, 3.28 \mathrm{mmol})$ was added. The mixture was heated for $7 \mathrm{~h}$ at $80^{\circ} \mathrm{C}$ and then cooled, quenched with water and extracted with $\mathrm{CH}_{2} \mathrm{Cl}_{2}(3 \times 30 \mathrm{ml})$. The organic phase was washed with water and dried over anhydrous $\mathrm{Na}_{2} \mathrm{SO}_{4}$. The solvent was evaporated in vacuo and the crude product was chromatographed (130 g silica gel, hexane/MTBE $=5: 1)$ to give $6(0.049 \mathrm{~g}, 4 \%)$ as dark red crystals and $7(1.005 \mathrm{~g}$, $88 \%)$ as deep red crystals.

Data of 6: ${ }^{1} \mathrm{H}$ NMR $\left(250 \mathrm{MHz}, \mathrm{CDCl}_{3}, 300 \mathrm{~K}\right): \delta=6.32(\mathrm{~s}, 1 \mathrm{H}, 11-\mathrm{H}), 5.12(\mathrm{~m}, 1 \mathrm{H}, 13-\mathrm{H}), 4.91(\mathrm{~m}, 1 \mathrm{H}, 16-\mathrm{H}), 4.34$ $(\mathrm{m}, 2 \mathrm{H}, 14-\mathrm{H}, 15-\mathrm{H}), 4.10(\mathrm{~s}, 5 \mathrm{H}, \mathrm{Cp}), 2.49(\mathrm{~d}, 1 \mathrm{H}, 4-\mathrm{H}, J=3.9 \mathrm{~Hz}), 1.97-2.11\left(\mathrm{~m}, 1 \mathrm{H}, 5-\mathrm{H}_{\text {exo }}\right), 1.63-1.72(\mathrm{~m}, 1 \mathrm{H}, 6-$ $\left.\mathrm{H}_{\text {exo }}\right), 1.39-1.50\left(\mathrm{~m}, 2 \mathrm{H}, 5-\mathrm{H}_{\text {endo }}, 6-\mathrm{H}_{\text {endo }}\right), 0.99(\mathrm{~s}, 3 \mathrm{H}, 10-\mathrm{H}), 0.94(\mathrm{~s}, 3 \mathrm{H}, 9-\mathrm{H}), 0.88(\mathrm{~s}, 3 \mathrm{H}, 8-\mathrm{H}) .{ }^{13} \mathrm{C} \mathrm{NMR}(62.90$ $\left.\mathrm{MHz}, \mathrm{CDCl}_{3}, 300 \mathrm{~K}\right): \delta=206.57$ (1C, 2-C), 138.17 (1C, 3-C), 132.47 (1C, 11-C), 78.77 (1C, 12-C), 72.21 (1C, 16-C), 71.60 (1C, 13-C), $70.56^{*}$ (1C, 14-C), $70.38^{*}$ (1C, 15-C), 69.13 (5C, Cp), 59.47 (1C, 1-C), 54.77 (1C, 4-C), 46.00 (1C, 7C), 29.83 (1C, 6-C), 27.51(1C, 5-C), 20.63 (1C, 8-C), 18.66 (1C, 9-C), 9.65 (1C, 10-C). MS (EI) m/z (rel. int.): 348 $\left(\mathrm{M}^{+}, 100\right), 283(36)$.

Data of 7: ${ }^{1} \mathrm{H}$ NMR $\left(250 \mathrm{MHz}, \mathrm{CDCl}_{3}, 300 \mathrm{~K}\right): \delta=7.04(\mathrm{~s}, 1 \mathrm{H}, 11-\mathrm{H}), 4.48-4.53(\mathrm{~m}, 2 \mathrm{H}, 13-\mathrm{H}, 16-\mathrm{H}), 4.36-4.41(\mathrm{~m}$, $2 \mathrm{H}, 14-\mathrm{H}, 15-\mathrm{H}), 4.13(\mathrm{~s}, 5 \mathrm{H}, \mathrm{Cp}), 2.93(\mathrm{~d}, 1 \mathrm{H}, 4-\mathrm{H}, J=4.2 \mathrm{~Hz}), 2.04-2.15\left(\mathrm{~m}, 1 \mathrm{H}, 5-\mathrm{H}_{\text {exo }}\right), 1.66-1.80\left(\mathrm{~m}, 1 \mathrm{H}, 6-\mathrm{H}_{\text {exo }}\right)$, 1.41-1.54 (m, 2H, 5- $\left.\mathrm{H}_{\text {endo }}, 6-\mathrm{H}_{\text {endo }}\right), 1.01(\mathrm{~s}, 3 \mathrm{H}, 10-\mathrm{H}), 0.99(\mathrm{~s}, 3 \mathrm{H}, 9-\mathrm{H}), 0.82(\mathrm{~s}, 3 \mathrm{H}, 8-\mathrm{H}) .{ }^{13} \mathrm{C} \mathrm{NMR}(62.90 \mathrm{MHz}$, $\left.\mathrm{CDCl}_{3}, 300 \mathrm{~K}\right): \delta=207.56$ (1C, 2-C), 138.51 (1C, 3-C), 127.79 (1C, 11-C), 78.86 (1C, 12-C), 71.29 (1C, 16-C), 70.43* (1C, 14-C), 69.31 (5C, Cp), 69.01* (1C, 15-C), 68.39 (1C, 13-C), 57.11 (1C, 1-C), 49.30 (1C, 4-C), 46.34 (1C, 7-C), 30.80 (1C, 6-C), 25.72 (1C, 5-C), 20.70 (1C, 8-C), 18.42 (1C, 9-C), 9.32 (1C, 10-C).

\section{3. \\ 1-((1S,4S,Z)-3-Ferrocenylmethylidene-7,7-dimethyl-2-oxobicyclo[2.2.1]heptan-1-yl)- $N, N$ - dimethylmethanesulfonamide 8 and 1-((1S,4S,E)-3-ferrocenylmethylidene-7,7-dimethyl-2- oxobicyclo[2.2.1]heptan-1-yl)- $N, N$-dimethylmethanesulfonamide 9}

A mixture of $3(0.50 \mathrm{~g}, 1.93 \mathrm{mmol}), \mathrm{KOH}(0.16 \mathrm{~g}, 2.89 \mathrm{mmol})$ and 18 -crown-6 (0.050 g, $0.19 \mathrm{mmol})$ in $12 \mathrm{ml}$ dry toluene was stirred for $30 \mathrm{~min}$ and $5(0.41 \mathrm{~g}, 1.93 \mathrm{mmol})$ was added. The mixture was heated for $5 \mathrm{~h}$ at $80^{\circ} \mathrm{C}$ (oil bath) and then cooled, quenched with water and extracted with $\mathrm{CH}_{2} \mathrm{Cl}_{2}(3 \times 30 \mathrm{ml})$. The combined organic layers were washed with water and dried over anhydrous $\mathrm{Na}_{2} \mathrm{SO}_{4}$. The solvent was evaporated in vacuo and the crude product was chromatographed (170 g silica gel, hexane/MTBE $=5: 1)$ to give $8(0.021 \mathrm{~g}, 2 \%)$ as dark red crystals and $9(0.799 \mathrm{~g}$, 91\%) as deep red crystals. Compounds $\mathbf{8}$ and $\mathbf{9}$ have identical physical and spectroscopic data as described in the literature. $^{58}$

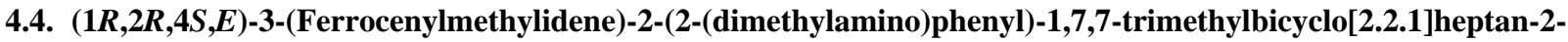
ol 15

To a solution of $n$-BuLi $(0.83 \mathrm{ml}, 1.32 \mathrm{mmol}$ of $1.6 \mathrm{M}$ solution in hexane) in $3 \mathrm{ml}$ of hexane were added TMED $(0.20 \mathrm{ml}, 1.32 \mathrm{mmol})$ and $N, N$-dimethylaniline $(0.20 \mathrm{ml}, 1.58 \mathrm{mmol})$. The mixture was refluxed for $4 \mathrm{~h}$ and after cooling to $0^{\circ} \mathrm{C}$ was added $7(0.23 \mathrm{~g}, 0.66 \mathrm{mmol})$ as a solid. The reaction mixture was stirred for $18 \mathrm{~h}$ (monitoring by TLC, petroleum ether/ $\mathrm{Et}_{2} \mathrm{O}=10: 1$ ), quenched with water, extracted with $\mathrm{Et}_{2} \mathrm{O}$ and the organic phase dried over anhydrous $\mathrm{Na}_{2} \mathrm{SO}_{4}$. The solvent was evaporated in vacuo and the crude product was chromatographed (70 g silica gel, 
petroleum ether/ $\left.\mathrm{Et}_{2} \mathrm{O}=40: 1\right)$, to give unreacted $7(0.085 \mathrm{~g}, 37 \%), 20(0.062 \mathrm{~g}, 20 \%)$ as a yellow oil and $15(0.053 \mathrm{~g}$, $17 \%$ ) as a yellow solid.

Data of 15: $\mathrm{Mp} 171-173^{\circ} \mathrm{C}$ (with decomp.). $[\alpha]_{\mathrm{D}}^{20}=-136.9\left(c 0.41, \mathrm{CHCl}_{3}\right) .{ }^{1} \mathrm{H} \mathrm{NMR}\left(250 \mathrm{MHz}, \mathrm{CDCl}_{3}, 300 \mathrm{~K}\right): \delta=$ $9.30(\mathrm{~s}, 1 \mathrm{H}, \mathrm{OH}), 7.40\left(\mathrm{dd}, 1 \mathrm{H}, 3^{`}-\mathrm{H}, J=8.0,1.4 \mathrm{~Hz}\right), 7.30-7.36\left(\mathrm{~m}, 1 \mathrm{H}, 6{ }^{-}-\mathrm{H}\right), 7.23-7.30$ (m, 1H, $\left.4 `-\mathrm{H}\right), 7.08-7.16$ (m, $1 \mathrm{H}, 5-\mathrm{H}), 6.10(\mathrm{~s}, 1 \mathrm{H}, 11-\mathrm{H}), 4.44(\mathrm{~m}, 1 \mathrm{H}, 16-\mathrm{H}), 4.28(\mathrm{~m}, 1 \mathrm{H}, 13-\mathrm{H}), 4.22(\mathrm{~m}, 1 \mathrm{H}, 15-\mathrm{H}), 4.16(\mathrm{~m}, 1 \mathrm{H}, 14-\mathrm{H}), 4.15(\mathrm{~s}$, $5 \mathrm{H}, \mathrm{Cp}), 2.88(\mathrm{~d}, 1 \mathrm{H}, 4-\mathrm{H}, J=4.3 \mathrm{~Hz}), 2.79^{*}\left(\mathrm{~s}, 3 \mathrm{H}, \mathrm{NMe}_{\mathrm{a}}\right), 2.71^{*}\left(\mathrm{~s}, 3 \mathrm{H}, \mathrm{NMe}_{\mathrm{b}}\right), 1.98\left(\mathrm{~m}, 1 \mathrm{H}, \mathrm{H}-5_{\text {exo }}\right), 1.58(\mathrm{~m}, 1 \mathrm{H}, \mathrm{H}-$ $\left.5_{\text {endo }}\right), 1.38\left(\mathrm{~m}, 1 \mathrm{H}, \mathrm{H}-6_{\text {exo }}\right), 1.11\left(\mathrm{~m}, 1 \mathrm{H}, \mathrm{H}-6_{\text {endo }}\right), 1.17(\mathrm{~s}, 3 \mathrm{H}, 8-\mathrm{H}), 0.96(\mathrm{~s}, 3 \mathrm{H}, 10-\mathrm{H}), 0.94(\mathrm{~s}, 3 \mathrm{H}, 9-\mathrm{H}) .{ }^{13} \mathrm{C} \mathrm{NMR}$ (62.90 MHz, CDCl3, $300 \mathrm{~K}): \delta=154.29$ (1C, $\left.2^{`}-\mathrm{C}\right), 152.03$ (1C, 3-C), 140.23 (1C, $\left.1^{`}-\mathrm{C}\right), 131.62$ (1C, $\left.6^{`}-\mathrm{C}\right), 127.76$ (1C, $\left.4^{`}-\mathrm{C}\right), 124.34$ (1C, 5-C), 123.53 (1C, 3`C), 122.78 (1C, 11-C), 89.13* (1C, 2-C), 83.78* (1C, 12-C), 69.61 (1C, 13-C), 68.55 (6C, 14-C, Cp), 68.22 (1C, 15-C), 67.62 (1C, 16C), 54.64 (1C, 1-C), 51.62 (1C, 4-C), 49.51 (1C, 7-C), 46.33* (1C, $\left.\mathrm{NMe}_{\mathrm{a}}\right), 49.03^{*}\left(1 \mathrm{C}, \mathrm{NMe}_{\mathrm{b}}\right), 31.15$ (1C, 6-C), 24.53 (1C, 5-C), 22.87 (1C, 8-C), 21.11 (1C, 10-C), 11.10 (1C, 9-C). MS (EI) $m / z$ (rel. int.): $469\left(\mathrm{M}^{+}, 100\right), 199$ (22), 167 (23), 149 (88). Anal. calcd. for $\mathrm{C}_{29} \mathrm{H}_{35} \mathrm{FeNO}$ (469.44) C, 74.20; H, 7.51; Fe, 11.90; N, 2.98. Found: C, 74.16; H, 7.59; Fe, 11.76; N, $2.89 \%$.

Data of 20: ${ }^{1} \mathrm{H}$ NMR $\left(250 \mathrm{MHz}, \mathrm{CDCl}_{3}, 300 \mathrm{~K}\right): \delta=7.08-7.27\left(\mathrm{~m}, 4 \mathrm{H}, 3-\mathrm{H}, 4-\mathrm{H}, 5^{-}-\mathrm{H}, 6-\mathrm{H}\right), 4.69$ (d, $1 \mathrm{H}, 3-\mathrm{H}, J=$ $11.8 \mathrm{~Hz}), 4.11(\mathrm{~m}, 1 \mathrm{H}, \mathrm{Fc}), 4.07$ (m, 3H, Fc), 3.77 (s, 5H, Cp), 2.69 (s, 6H, NMe $), 2.23$ (d, 1H, 11-H, J = 11.8 Hz), 2.09 $(\mathrm{d}, 1 \mathrm{H}, 4-\mathrm{H}, J=4.1 \mathrm{~Hz}), 1.93(\mathrm{~m}, 1 \mathrm{H}), 1.53(\mathrm{~m}, 1 \mathrm{H}), 1.22-1.36(\mathrm{~m}, 1 \mathrm{H}), 1.16(\mathrm{~s}, 3 \mathrm{H}, \mathrm{Me}), 0.89(\mathrm{~s}, 3 \mathrm{H}, \mathrm{Me}), 0.85(\mathrm{~m}$, $1 \mathrm{H}), 0.78(\mathrm{~s}, 3 \mathrm{H}, \mathrm{Me}) .{ }^{13} \mathrm{C} \mathrm{NMR}\left(\mathrm{CDCl}_{3}, 300 \mathrm{~K}\right): \delta=217.22(1 \mathrm{C}, \mathrm{C}=\mathrm{O}), 151.76(1 \mathrm{C}$, quaternary $\mathrm{Ar}), 142.80(1 \mathrm{C}$, quaternary Ar), 128.04 (1C, $\mathrm{CH} \mathrm{Ar}), 126.54$ (1C, $\mathrm{CH} \mathrm{Ar}), 124.06$ (1C, $\mathrm{CH} \mathrm{Ar}), 120.12$ (1C, CH Ar), 94.87 (1C, quaternary), 70.27 (1C, Fc), 68.59 (5C, Cp), 67.84 (1C, Fc), 66.87 (1C, Fc), 65.33 (1C, Fc), 61.60 (1C, CH), 57.99 (1C, quaternary), $47.13(1 \mathrm{C}, \mathrm{CH}), 45.95$ (1C, quaternary), $45.41\left(2 \mathrm{C}, \mathrm{NMe}_{2}\right), 38.45(1 \mathrm{C}, \mathrm{CH}), 29.79\left(1 \mathrm{C}, \mathrm{CH}_{2}\right), 29.26(1 \mathrm{C}$, $\left.\mathrm{CH}_{2}\right), 20.83$ (1C, Me), 20.67 (1C, Me), 9.47 (1C, Me).

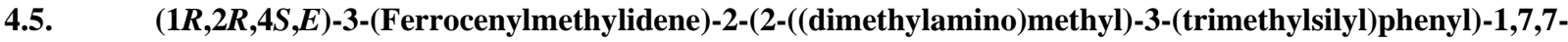 trimethylbicyclo[2.2.1]heptan-2-ol 16}

To a solution of $n$-BuLi $(2.14 \mathrm{ml}, 3.42 \mathrm{mmol}$ of $1.6 \mathrm{M}$ solution in hexane) in $6 \mathrm{ml}$ of hexane were added $1 \mathrm{ml}$ of $\mathrm{Et}_{2} \mathrm{O}$ and $\mathrm{N}, \mathrm{N}$-dimethyl(2-(trimethylsilyl)phenyl)methanamine $(0.71 \mathrm{~g}, 3.42 \mathrm{mmol})$ at room temperature. The solution was refluxed for $3 \mathrm{~h}$ and its color changed from colorless to a clear deep red. The mixture was then cooled to $0^{\circ} \mathrm{C}$ and 7 was added $(0.30 \mathrm{~g}, 0.86 \mathrm{mmol})$ as a solid (reaction progress was monitored by TLC, petroleum ether/Et $\left.\mathrm{O}_{2} \mathrm{O}=5: 1\right)$. After stirring for $1 \mathrm{~h}$ at $0^{\circ} \mathrm{C}$, it was quenched with water, extracted with $\mathrm{Et}_{2} \mathrm{O}$ and the organic phase was dried over anhydrous $\mathrm{Na}_{2} \mathrm{SO}_{4}$. The solvent was evaporated in vacuo and the crude product was chromatographed $(70 \mathrm{~g}$ silica gel, petroleum ether $/ \mathrm{Et}_{2} \mathrm{O}=20: 1$ to $\left.3: 1\right)$ to give $16(0.09 \mathrm{~g}, 19 \%)$ as an orange-yellow solid. $\mathrm{Mp} 81-83^{\circ} \mathrm{C}$. $[\alpha]_{\mathrm{D}}^{20}=-381.8(c 0.40$, $\mathrm{CHCl}_{3}$ ). ${ }^{1} \mathrm{H}$ NMR $\left(250 \mathrm{MHz}, \mathrm{CDCl}_{3}, 300 \mathrm{~K}\right): \delta=10.07$ (br s, $\left.1 \mathrm{H}, \mathrm{OH}\right), 7.69$ (dd, $\left.1 \mathrm{H}, 6^{\circ}-\mathrm{H}, J=8.0,2.4 \mathrm{~Hz}\right), 7.41(\mathrm{dd}$, $\left.1 \mathrm{H}, 4^{`}-\mathrm{H}, J=7.3,1.5 \mathrm{~Hz}\right), 7.18\left(\mathrm{t}, 1 \mathrm{H}, 5^{`}-\mathrm{H}, J=7.6 \mathrm{~Hz}\right), 6.18(\mathrm{~s}, 1 \mathrm{H}, 11-\mathrm{H}), 4.55\left(\mathrm{~d}, 1 \mathrm{H}, 7^{`}-\mathrm{H}_{\mathrm{a}}, J=12.1 \mathrm{~Hz}\right), 4.48(\mathrm{~m}$, $1 \mathrm{H}, 16-\mathrm{H}), 4.31(\mathrm{~m}, 1 \mathrm{H}, 13-\mathrm{H}), 4.24(\mathrm{~m}, 1 \mathrm{H}, 15-\mathrm{H}), 4.17-4,21(\mathrm{~m}, 1 \mathrm{H}, 14-\mathrm{H}), 4.19(\mathrm{~s}, 5 \mathrm{H}, \mathrm{Cp}), 3.38\left(\mathrm{~d}, 1 \mathrm{H}, 7-\mathrm{H}_{\mathrm{b}}, J=\right.$ $12.1 \mathrm{~Hz}), 2.86(\mathrm{~d}, 1 \mathrm{H}, 4-\mathrm{H}, J=4.4 \mathrm{~Hz}), 2.18\left(\mathrm{~s}, 6 \mathrm{H}, \mathrm{NMe}_{2}\right), 1.81\left(\mathrm{tt}, 1 \mathrm{H}, 6-\mathrm{H}_{\text {exo }}, J=12.1,4.1 \mathrm{~Hz}\right), 1.43$ (ddd, $1 \mathrm{H}, 6-$ $\left.\mathrm{H}_{\text {endo }}, J=13.1,9.2,4.6 \mathrm{~Hz}\right), 1.10-1.26\left(\mathrm{~m}, 1 \mathrm{H}, 5-\mathrm{H}_{\text {exo }}\right), 1.17(\mathrm{~s}, 3 \mathrm{H}, 8-\mathrm{H}), 1.02(\mathrm{~s}, 3 \mathrm{H}, 10-\mathrm{H}), 0.89(\mathrm{~s}, 3 \mathrm{H}, 9-\mathrm{H}), 0.43$ (ddd, $\left.1 \mathrm{H}, 5-\mathrm{H}_{\text {endo }}, J=13.1,8.8,4.0 \mathrm{~Hz}\right), 0.37$ (s, 9H, $\left.\mathrm{SiMe}_{3}\right) .{ }^{13} \mathrm{C} \mathrm{NMR}\left(62.90 \mathrm{MHz}, \mathrm{CDCl}_{3}, 300 \mathrm{~K}\right): \delta=155.28(1 \mathrm{C}, 3-$

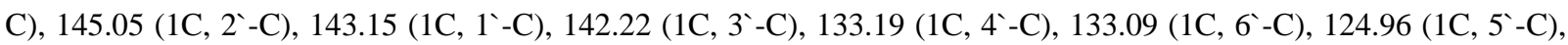
121.21 (1C, 11-C), 88.28* (1C, 2-C), 84.35* (1C, 12-C), 69.44 (1C, 13-C), 68.51 (5C, Cp), 68.12 (1C, 14-C), 68.09 (1C, 15-C), 67.69 (1C, 16-C), 62.95 (1C, 7`-C), 55.87 (1C, 1-C), 51.82 (1C, 4-C), 49.42 (1C, 7-C), 43.03 (2C, $\left.\mathrm{NMe}_{2}\right), 30.77$ (1C, 5-C), 23.26 (1C, 6-C), 22.59 (1C, 8-C), 21.29 (1C, 9-C), 12.51 (1C, 10-C), 2.19 (3C, SiMe $)_{3} \mathrm{MS}(\mathrm{EI}) \mathrm{m} / z(\mathrm{rel}$. int.): $555\left(\mathrm{M}^{+}, 5\right), 167$ (21), 149 (100), 129 (29). Anal. calcd. for $\mathrm{C}_{33} \mathrm{H}_{45} \mathrm{FeNOSi}$ (555.65): C, 71.33; H, 8.16; Fe, 10.05; N, 2.52. Found: C, 71.45; H, 8.24; Fe, 10.16; N, $2.63 \%$.

4.6.

$(1 R, 2 R, 4 S, E)-3-(F e r r o c e n y l m e t h y l i d e n e)-2-(2-((d i m e t h y l a m i n o) m e t h y l) p h e n y l)-1,7,7-$ trimethylbicyclo[2.2.1]heptan-2-ol 17

To a solution of $N, N$-dimethylbenzylamine $(0.31 \mathrm{~g}, 2.25 \mathrm{mmol})$ in $4 \mathrm{ml}$ of $\mathrm{Et}_{2} \mathrm{O}$ was added $n$-BuLi $(1.70 \mathrm{ml}$, $2.70 \mathrm{mmol}$ of $1.6 \mathrm{M}$ solution in hexane) at room temperature and stirred for $48 \mathrm{~h}$; next $5 \mathrm{ml}$ of $\mathrm{Et}_{2} \mathrm{O}$ and 7 (0.50 g, 1.44 mmol) were added at room temperature. The reaction mixture was monitored by TLC (petroleum ether/Et ${ }_{2} \mathrm{O} / \mathrm{Et}_{3} \mathrm{~N}=$ 100:20:1) and after $1 \mathrm{~h}$ was quenched at $0^{\circ} \mathrm{C}$ with $8 \%$ aqueous $\mathrm{H}_{3} \mathrm{PO}_{4}(\mathrm{pH} 4)$, and extracted with $\mathrm{Et}_{2} \mathrm{O}$ to remove 7 . The aqueous phase was alkalized with solid $\mathrm{K}_{2} \mathrm{CO}_{3}$ to $\mathrm{pH} 11$, extracted with $\mathrm{Et}_{2} \mathrm{O}$ and the organic phase was dried over anhydrous $\mathrm{Na}_{2} \mathrm{SO}_{4}$. The solvent was evaporated in vacuo and the crude product was chromatographed (87 g silica gel, $\left.\mathrm{Et}_{2} \mathrm{O}\right)$ to give $17(0.23 \mathrm{~g}, 33 \%)$ as an orange solid. $\mathrm{Mp} 66-68^{\circ} \mathrm{C} .[\alpha]_{\mathrm{D}}^{20}=-389.7\left(c 1.00, \mathrm{CHCl}_{3}\right) .{ }^{1} \mathrm{H} \mathrm{NMR}(250 \mathrm{MHz}$, $\left.\mathrm{CDCl}_{3}, 300 \mathrm{~K}\right): \delta=9.03($ br s, $1 \mathrm{H}, \mathrm{OH}), 7.57\left(\mathrm{dd}, 1 \mathrm{H}, 6^{`}-\mathrm{H}, J=16.9,1.5 \mathrm{~Hz}\right), 7.12-7.25$ (m, 3H, $\left.3^{`}-\mathrm{H}, 4^{`}-\mathrm{H}, 5^{`}-\mathrm{H}\right), 6.09$ $(\mathrm{s}, 1 \mathrm{H}, 11-\mathrm{H}), 4.54\left(\mathrm{~d}, 1 \mathrm{H}, 7-\mathrm{H}_{\mathrm{a}}, J=11.7 \mathrm{~Hz}\right), 4.46(\mathrm{~m}, 1 \mathrm{H}, 16-\mathrm{H}), 4.29(\mathrm{~m}, 1 \mathrm{H}, 13-\mathrm{H}), 4.23(\mathrm{~m}, 1 \mathrm{H}, 15-\mathrm{H}), 4.18(\mathrm{~s}$, $5 \mathrm{H}, \mathrm{Cp}), 4.15-4.20(\mathrm{~m}, 1 \mathrm{H}, 14-\mathrm{H}), 2.87(\mathrm{~d}, 1 \mathrm{H}, \mathrm{H}-4, J=4.4 \mathrm{~Hz}), 2.87\left(\mathrm{~d}, 1 \mathrm{H}, 7-\mathrm{H}_{\mathrm{b}}, J=11.7 \mathrm{~Hz}\right), 2.24\left(\mathrm{~s}, 6 \mathrm{H}, \mathrm{NMe}_{2}\right)$, 
$2.20(\mathrm{~m}, 1 \mathrm{H}, 4-\mathrm{H}), 1.89\left(\mathrm{~m}, 1 \mathrm{H}, 6-\mathrm{H}_{\text {exo }}\right), 1.52\left(\mathrm{ddd}, 1 \mathrm{H}, 6-\mathrm{H}_{\text {endo }}, J=12.0,8.1,4.7 \mathrm{~Hz}\right), 1.29$ (ddd, 1H, 5- $\mathrm{H}_{\text {exo }}, J=12.0$, 4.7, $1.3 \mathrm{~Hz}) 1.16(\mathrm{~s}, 3 \mathrm{H}, 9-\mathrm{H}), 1.07(\mathrm{~s}, 3 \mathrm{H}, 10-\mathrm{H}), 0.91(\mathrm{~s}, 3 \mathrm{H}, 8-\mathrm{H}), 0.78-0.90\left(\mathrm{~m}, 1 \mathrm{H}, \mathrm{H}-5_{\text {endo }}\right) .{ }^{13} \mathrm{C} \mathrm{NMR}(62.90 \mathrm{MHz}$, $\left.\mathrm{CDCl}_{3}, 300 \mathrm{~K}\right): \delta=154.91$ (1C, 3-C), 145.99* (1C, $\left.2^{`}-\mathrm{C}\right), 136.74^{*}$ (1C, 1`-C), 133.37 (1C, $\left.5 ` \mathrm{C}\right), 132.07$ (1C, $\left.6^{`}-\mathrm{C}\right)$, 126.39 (1C, $\left.33^{`} \mathrm{C}\right), 126.22$ (1C, $\left.4^{-}-\mathrm{C}\right), 121.60$ (1C, $\left.11-\mathrm{C}\right), 88.64^{*}$ (1C, 2-C), 84.21* (1C, 12-C), 69.45 (1C, 13-C), 68.51 (5C, Cp), 68.11* (1C, 15-C), 67.64 (1C, 16-C), 67.64* (1C, 14-C), 65.13 (1C, 7`-C), 55.38 (1C, 1-C), 51.72 (1C, 4-C), 49.60 (1C, 7-C), 44.10 (2C, $\mathrm{NMe}_{2}$ ), 31.04 (1C, 5-C), 23.57 (1C, 6-C), 22.52 (1C, 9-C), 21.22 (1C, 8-C), 12.86 (1C, 10C). MS (EI) $\mathrm{m} / z$ (rel. int.): $483\left(\mathrm{M}^{+\bullet}, 5\right), 149$ (100), 129 (19). Anal. calcd. for $\mathrm{C}_{30} \mathrm{H}_{37} \mathrm{FeNO}$ (483.47): C, 74.53; H, 7.71; Fe, 11.55; N, 2.90. Found: C, 74.67; H, 7.80; Fe, 11.41; N, $2.79 \%$.

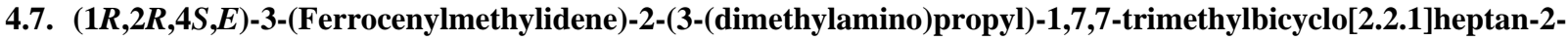
ol 18

Method A:

To a solution of $7(0.36 \mathrm{~g}, 1.03 \mathrm{mmol})$ in $20 \mathrm{ml}$ of hexane was added $\mathbf{1 3}$ as a solid $(0.14 \mathrm{~g}, 1.55 \mathrm{mmol})$ at room temperature. The reaction was monitored by TLC (petroleum ether/ $\mathrm{Et}_{2} \mathrm{O}=1: 1$ ) and after stirring for $1 \mathrm{~h}$ at room temperature, was quenched with water, extracted with $\mathrm{Et}_{2} \mathrm{O}$, and the organic phase was dried over anhydrous $\mathrm{Na}_{2} \mathrm{SO}_{4}$. The solvent was evaporated in vacuo and the crude product was chromatographed (79 $\mathrm{g}$ silica gel, $\left.\mathrm{Et}_{2} \mathrm{O} / \mathrm{Et}_{3} \mathrm{~N}=50: 1\right)$ to give $21(0.18 \mathrm{~g}, 40 \%)$ as an orange oil (diastereomeric mixture of 3 isomers in ratio 5:10:12) and pure $18(0.02 \mathrm{~g}, 5 \%)$ as an orange-yellow solid.

Method B:

A mixture of $\mathrm{CeCl}_{3}(0.14 \mathrm{~g}, 0.57 \mathrm{mmol})$ and $5 \mathrm{ml}$ of $\mathrm{THF}$ was stirred at room temperature under an argon atmosphere for $15 \mathrm{~min}$. Ketone $7(0.20 \mathrm{~g}, 0.57 \mathrm{mmol})$ was added as a solid and the mixture was stirred for an additional $1 \mathrm{~h}$. To this mixture was added 13 as a solid $(0.11 \mathrm{~g}, 1.15 \mathrm{mmol})$. The reaction was monitored by $\mathrm{TLC}\left(\mathrm{Et}_{2} \mathrm{O} / \mathrm{Et}_{3} \mathrm{~N}=\right.$ 200:1) and after $1 \mathrm{~h}$ it was quenched with diluted aqueous $\mathrm{NH}_{4} \mathrm{Cl}$, and extracted with $\mathrm{Et}_{2} \mathrm{O}$. The organic phase was dried over anhydrous $\mathrm{Na}_{2} \mathrm{SO}_{4}$, the solvent was evaporated in vacuo and the crude product was chromatographed (115 g silica gel, petroleum ether/ $\mathrm{Et}_{2} \mathrm{O}=1: 1$ then $\left.\mathrm{Et}_{2} \mathrm{O} / \mathrm{Et}_{3} \mathrm{~N}=200: 1\right)$ to give $21(0.06 \mathrm{~g}, 22 \%$, diastereomeric mixture of two isomers in a ratio 2:5, major and minor respectively) as an orange oil, and pure $\mathbf{1 8}(0.13 \mathrm{~g}, 53 \%)$ as an orange-yellow solid.

Data of 18: $\mathrm{Mp} 102-103^{\circ} \mathrm{C} .[\alpha]_{\mathrm{D}}^{20}=-31.0\left(c 0.58, \mathrm{CHCl}_{3}\right) .{ }^{1} \mathrm{H} \mathrm{NMR}\left(250 \mathrm{MHz}, \mathrm{CDCl}_{3}, 300 \mathrm{~K}\right): \delta=6.09(\mathrm{~s}, 1 \mathrm{H}, 11-\mathrm{H})$, $4.31(\mathrm{~m}, 1 \mathrm{H}, 16-\mathrm{H}), 4.28(\mathrm{~m}, 1 \mathrm{H}, 13-\mathrm{H}), 4.12-4.19$ (m, 2H, 14-H, 15-H), 4.10 (s, 5H, Cp), 2.73 (d, 1H, 4-H, J=4.9 Hz), $2.28\left(\mathrm{~s}, 6 \mathrm{H}, \mathrm{NMe}_{2}\right)$ 2.20-2.40 (m, 2H, 3`- $\left.\mathrm{H}\right), 1.84-1.97\left(\mathrm{~m}, 1 \mathrm{H}, 5-\mathrm{H}_{\text {exo }}\right), 1.60-1.90(\mathrm{~m}, 2 \mathrm{H}, 1 `-\mathrm{H}), 1.60-1.84(\mathrm{~m}, 2 \mathrm{H}, 2 `$ $\mathrm{H}), 1.50-1.62\left(\mathrm{~m}, 2 \mathrm{H}, 6-\mathrm{H}_{\text {exo }}, 6-\mathrm{H}_{\text {endo }}\right), 1.20-1.31\left(\mathrm{~m}, 1 \mathrm{H}, 5-\mathrm{H}_{\text {endo }}\right), 1.01(\mathrm{~s}, 3 \mathrm{H}, 8-\mathrm{H}), 0.99(\mathrm{~s}, 3 \mathrm{H}, 10-\mathrm{H}), 0.88(\mathrm{~s}, 3 \mathrm{H}, 9-$ H). ${ }^{13} \mathrm{C}$ NMR $\left(62.90 \mathrm{MHz}, \mathrm{CDCl}_{3}, 300 \mathrm{~K}\right): \delta=154.62$ (1C, 3-C), 116.28 (1C, 11-C), 83.68* $(1 \mathrm{C}, 2-\mathrm{C}), 80.18^{*}(1 \mathrm{C}, 12-$ C), $69.02(1 \mathrm{C}, 13-\mathrm{C}), 68.85$ (5C, Cp), 68.12 (1C, 14-C), $68.12(1 \mathrm{C}, 15-\mathrm{C}), 68.05$ (1C, 16-C), 60.85 (1C, 3-C), 52.54 (1C, 1-C), 50.97 (1C, 4-C), 49.17 (1C, 7-C), 45.62 (2C, $\mathrm{NMe}_{2}$ ), 40.62 (1C, 1 - $\left.-\mathrm{C}\right), 30.79$ (1C, 6-C), 25.37 (1C, 5-C), 23.26 (1C, 2 -C), 22.39 (1C, 8-C), 20.10 (1C, 9-C), 12.11 (1C, 10-C). MS (EI) m/z (rel. int.): 435 (M M $\left.^{+\bullet} 100\right), 364$ (21), 283 (23), 199 (16), 149 (22). Anal. calcd. for $\mathrm{C}_{26} \mathrm{H}_{37} \mathrm{FeNO}$ (435.42): C, 71.72; H, 8.56; Fe, 12.83; N, 3.22. Found: C, $71.77 ; \mathrm{H}, 8.67 ; \mathrm{Fe}, 13.00 ; \mathrm{N}, 3.31 \%$.

Data of 21 (data extracted from a mixture of two isomers, obtained according Method B): ${ }^{1} \mathrm{H} \mathrm{NMR} \mathrm{(250} \mathrm{MHz} \mathrm{CDCl}_{3}$, $300 \mathrm{~K}): \delta=4.13$ (s, Cp minor isomer), 4.11 (s, Cp major isomer), 4.06-4.11 (m), 4.02-4.05 (m), 3.93-3.98 (m), 2.56$2.69(\mathrm{~m}), 2.20-2.35(\mathrm{~m}), 2.24\left(\mathrm{~s}, \mathrm{NMe}_{2}\right), 2.10-2.18(\mathrm{~m}), 1.15-1.98(\mathrm{~m}), 0.97$ (s, Me major isomer), 0.89 (s, Me major isomer), 0.86 (s, Me minor isomer), 0.85 (s, Me minor isomer), 0.84 (s, Me minor isomer) 0.83 (s, Me major isomer). MS (EI) $m / z$ (rel. int.): 435 (M+ $\left.\mathrm{M}^{+}, 100\right), 362$ (15), 336 (16), 237 (18), 171 (9), 121 (15), 58 (42).

\section{8. (1R,2R,4S,E)-3-(Ferrocenylmethylidene)-1,7,7-trimethyl-2-(thiophen-2-yl)bicyclo[2.2.1]heptan-2-ol (19)}

Method A:

To a mixture of $n$-BuLi $(0.77 \mathrm{ml}, 0.92 \mathrm{mmol}$ of $1.6 \mathrm{M}$ solution in hexane) and $1.0 \mathrm{ml}$ THF was added thiophene $(0.07 \mathrm{ml}, 0.92 \mathrm{mmol})$ at $0^{\circ} \mathrm{C}$. The light yellow solution was allowed to warm to room temperature and stirred for $1 \mathrm{~h}$, then cooled down to $-50^{\circ} \mathrm{C}$ and $7(0.16 \mathrm{~g}, 0.46 \mathrm{mmol})$ was added as a solid. The mixture was stirred and allowed to warm slowly to room temperature; the reaction progress was monitored by TLC (petroleum ether/ $\mathrm{Et}_{2} \mathrm{O}=5: 1$ ). After $1.5 \mathrm{~h} 7$ was consumed completely and the mixture was quenched with water, extracted with $\mathrm{Et}_{2} \mathrm{O}$, and the organic phase was dried over anhydrous $\mathrm{Na}_{2} \mathrm{SO}_{4}$. The solvent was evaporated in vacuo and the crude product was chromatographed $\left(110 \mathrm{~g}\right.$ silica gel, petroleum ether/toluene/ $\mathrm{Et}_{2} \mathrm{O}=50: 1: 1$ then petroleum ether/toluene $\left.=15: 1\right)$ to give pure $19(0.021 \mathrm{~g}$, $8 \%)$ as an orange solid and $0.071 \mathrm{~g}(28 \%)$ mixture of $\mathbf{1 9}$ and $\mathbf{2 2}$ (22 as a mixture of two isomers).

Method B: 
To a mixture of $n$-BuLi (1.00 ml, $1.2 \mathrm{mmol}$ of $1.2 \mathrm{M}$ solution in hexane) and $1.0 \mathrm{ml}$ THF was added thiophene $(0.077 \mathrm{~g}, 0.07 \mathrm{ml}, 0.92 \mathrm{mmol})$ at $0^{\circ} \mathrm{C}$. The formed light yellow solution of 2-lithiothiophene was allowed to warm to room temperature and stirred for $1 \mathrm{~h}$. Separately prepared was a suspension of $\mathrm{CeCl}_{3}(0.11 \mathrm{~g}, 0.46 \mathrm{mmol})$ in $2 \mathrm{ml} \mathrm{THF}$ and $7(0.16 \mathrm{~g}, 0.46 \mathrm{mmol})$, and it was stirred at room temperature under argon within 1.5. The mixture was then cooled to $-40^{\circ} \mathrm{C}$ and the solution of 2-lithiothiophene was added. The mixture was allowed to warm slowly to room temperature. The reaction progress was monitored by TLC (petroleum ether/ $\mathrm{Et}_{2} \mathrm{O}=5: 1$ ). After stirring for $20 \mathrm{~h}$ at room temperature, 7 was converted completely and the mixture was quenched with saturated aqueous $\mathrm{NH}_{4} \mathrm{Cl}$, extracted with $\mathrm{Et}_{2} \mathrm{O}$ and the organic phase was dried over anhydrous $\mathrm{Na}_{2} \mathrm{SO}_{4}$. The solvent was evaporated in vacuo and the crude product was chromatographed ( $37 \mathrm{~g}$ silica gel, petroleum ether/ $\left.\mathrm{Et}_{2} \mathrm{O}=30: 1\right)$ to give pure $19(0.10 \mathrm{~g}, 50 \%)$ as orange solid.

Data of 19: Mp 113-114 ${ }^{\circ} \mathrm{C} .[\alpha]_{\mathrm{D}}^{20}=-261.0$ (c 1.29, hexane). ${ }^{1} \mathrm{H}$ NMR $\left(250 \mathrm{MHz}, \mathrm{CDCl}_{3}, 300 \mathrm{~K}\right): \delta=7.26\left(\mathrm{dd}, 1 \mathrm{H}, 2^{`}-\mathrm{H}\right.$, $J=4.9,1.5 \mathrm{~Hz}), 6.97(\mathrm{~d}, 1 \mathrm{H}, 4-\mathrm{H}, J=7.0 \mathrm{~Hz}), 6.97-7.03(\mathrm{~m}, 1 \mathrm{H}, 3-\mathrm{H}), 6.30(\mathrm{~s}, 1 \mathrm{H}, 11-\mathrm{H}), 4.42(\mathrm{~m}, 1 \mathrm{H}, 16-\mathrm{H}), 4.34$ $(\mathrm{m}, 1 \mathrm{H}, 15-\mathrm{H}), 4.26(\mathrm{~m}, 1 \mathrm{H}, 14-\mathrm{H}), 4.23(\mathrm{~m}, 1 \mathrm{H}, 13-\mathrm{H}), 4.20(\mathrm{~s}, 5 \mathrm{H}, \mathrm{Cp}), 2.92(\mathrm{~d}, 1 \mathrm{H}, 4-\mathrm{H}, J=4.4 \mathrm{~Hz}), 2.26(\mathrm{~s}, 1 \mathrm{H}$, $\mathrm{OH}), 1.92\left(\mathrm{~m}, 1 \mathrm{H}, 5-\mathrm{H}_{\text {exo }}\right), 1.35-1.46\left(\mathrm{~m}, 2 \mathrm{H}, 5-\mathrm{H}_{\text {endo }}, 6-\mathrm{H}_{\text {exo }}\right), 1.00-1.08\left(\mathrm{~m}, 1 \mathrm{H}, 6-\mathrm{H}_{\text {endo }}\right), 1.15(\mathrm{~s}, 3 \mathrm{H}, 8-\mathrm{H}), 1.10(\mathrm{~s}, 3 \mathrm{H}$, $10-\mathrm{H}), 0.98(\mathrm{~s}, 3 \mathrm{H}, 9-\mathrm{H}) .{ }^{13} \mathrm{C}$ NMR $\left(62.90 \mathrm{MHz}, \mathrm{CDCl}_{3}, 300 \mathrm{~K}\right): \delta=151.71(1 \mathrm{C}, 3-\mathrm{C}), 151.00$ (1C, $\left.1^{`}-\mathrm{C}\right), 125.86(1 \mathrm{C}$, 4 -C), 125.49 (1C, 3 - $-\mathrm{C}), 124.65$ (1C, 2`-C), 121.85 (1C, 11-C), 83.74* (1C, 2-C), 82.67 (1C, 12-C), 68.79 (5C, Cp), 68.55 (2C, 13-C, 14-C), 69.26 (1C, 15-C), 68.05 (1C, 16-C), 54.40 (1C, 1-C), 51.24 (1C, 4-C), 48.77 (1C, 7-C), 31.54 (1C, 6-C), 24.59 (1C, 5-C), 22.42 (1C, 8-C), 20.90 (1C, 9-C), 9.64 (1C, 10-C). MS (EI) m/z (rel. int.): 432 (M ( $\left.^{+}, 100\right)$, 416 (13), 149 (71). Anal. calcd. for $\mathrm{C}_{25} \mathrm{H}_{28} \mathrm{FeOS}$ (432.40): C, 69.44; H, 6.53; Fe, 12.92; S, 7.42. Found: C, 69.60; H, $6.79 ; \mathrm{Fe}, 13.09 ; \mathrm{S}, 7.32 \%$.

Data of 22 (data extracted from a mixture of 19 and 22, obtained according Method A): ${ }^{1} \mathrm{H}$ NMR $\left(250 \mathrm{MHz}, \mathrm{CDCl}_{3}\right.$, $300 \mathrm{~K}): \delta=7.72-7.71$ (m, 22-Ar), 7.56-7.50 (m, 22-Ar), 7.26-7.23 (m, 19-Ar), 7.22-7.20 (m, 22-Ar), 7.01-6.92 (m, 19Ar, 22-Ar), 6.28 (s, 19), 4.40-4.39 (m, 19-Fc), 4.33-4.31 (m, 19-Fc), 4.24-4.20 (m, 19-Fc, 22), 4.18 (s, 22-Fc), 4-16-4.10 (m, 22-Fc), 3.97 (d, 22), 2.89 (d, 19), 2.80-2.42 (m, 22), 1.96-1.84 (m, 19), 1.71-1.64 (m, 22), 1.45-1.27 (m, 19, 22), 1.13 (s, 19), 1.09 (s, 19), 0.97-0.82 (m, 19, 22).

4.9.

1-((1S,2R,4S,E)-3-(Ferrocenylmethylidene)-2-(3-(dimethylamino)propyl)-2-hydroxy-7,7dimethylbicyclo[2.2.1] heptan-1-yl)- $N, N$-dimethylmethanesulfonamide 29

To a solution of 3-(dimethylamino)propyllithium $(0.43 \mathrm{~g}, 4.62 \mathrm{mmol})$ in $5 \mathrm{ml}$ toluene was added $9(0.70 \mathrm{~g}$, $1.54 \mathrm{mmol})$ at $-50^{\circ} \mathrm{C}$. After stirring at this temperature for $3 \mathrm{~h}$, the mixture was allowed to warm to $5^{\circ} \mathrm{C}$. The reaction progress was monitored by TLC (EtOAc/MeOH/ $\mathrm{CH}_{2} \mathrm{Cl}_{2} / \mathrm{Et}_{3} \mathrm{~N}=$ 5.8:0.05:0.05:0.1) until 9 was consumed. The mixture was quenched with water $(10 \mathrm{ml})$, extracted with $\mathrm{Et}_{2} \mathrm{O}(3 \times 20 \mathrm{ml})$ and the organic phase was dried over anhydrous $\mathrm{Na}_{2} \mathrm{SO}_{4}$. The solvent was evaporated in vacuo and the crude product was chromatographed (90 g silica gel, $\mathrm{EtOAc} / \mathrm{MeOH} / \mathrm{CH}_{2} \mathrm{Cl}_{2} / \mathrm{Et}_{3} \mathrm{~N}=$ 5.8:0.05:0.05:0.001) to give $36(0.413 \mathrm{~g}, 48 \%$; diastereomeric mixture of two isomers in ratio 1:1) as an orange-yellow oil and pure $29(0.306 \mathrm{~g}, 37 \%)$ as an orange-yellow solid.

Data of 29: Mp 137-139 ${ }^{\circ} \mathrm{C} .[\alpha]_{\mathrm{D}}^{20}=-68.3\left(c 0.38, \mathrm{CHCl}_{3}\right) .{ }^{1} \mathrm{H} \mathrm{NMR}\left(600 \mathrm{MHz}, \mathrm{CDCl}_{3}, 293 \mathrm{~K}\right): \delta=6.17(\mathrm{~s}, 1 \mathrm{H}, 11-\mathrm{H})$, 4.30-4.29 (m, 1H, 16-H), 4.28-4.27 (m, 1H, 13-H), 4.19-4.18* (m, 1H, 14-H), 4.17-4.16* (m, 1H, 15-H), 4.10 (s, 5H, $\mathrm{Cp}), 3.47\left(\mathrm{~d}, 1 \mathrm{H}, 10-\mathrm{H}_{\mathrm{a}}, J=14.3 \mathrm{~Hz}\right), 2.90\left(\mathrm{~s}, 6 \mathrm{H}, \mathrm{SO}_{2} \mathrm{NMe}_{2}\right), 2.74(\mathrm{~d}, 1 \mathrm{H}, 4-\mathrm{H}, J=4.9 \mathrm{~Hz}) 2.68\left(\mathrm{~d}, 1 \mathrm{H}, 10-\mathrm{H}_{\mathrm{b}}, J=14.3\right.$

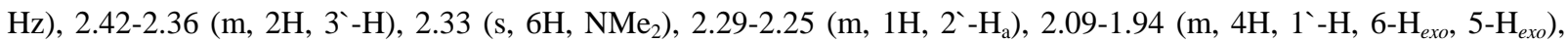
1.80-1.73 (m, 2H, 2- $\left.-\mathrm{H}_{\mathrm{b}}, 5-\mathrm{H}_{\text {endo }}\right), 1.40-1.35\left(\mathrm{~m}, 1 \mathrm{H}, 6-\mathrm{H}_{\text {endo }}\right), 1.01(\mathrm{~s}, 3 \mathrm{H}, 8-\mathrm{H}), 0.96(\mathrm{~s}, 3 \mathrm{H}, 9-\mathrm{H}) .{ }^{13} \mathrm{C} \mathrm{NMR}(150.92$ $\left.\mathrm{MHz}, \mathrm{CDCl}_{3}, 293 \mathrm{~K}\right): \delta=152.56$ (1C, 3-C), 116.72 (1C, 11-C), 83.44 (1C, 2-C), 78.84 (1C, 12-C), 69.01 (1C, 13-C), 68.83 (5C, Cp), 68.21 (1C, 16-C), 68.13 (2C, 14-C, 15-C), 60.63 (1C, 3`-C), 53.98 (1C, 1-C), 50.69 (1C, 7-C), 50.45 (1C, 4-C), 45.43 (2C, $\mathrm{NMe}_{2}$ ), 44.39 (1C, 10-C), 38.47 (1C, 2`C), 37.69 (2C, $\mathrm{SO}_{2} \mathrm{NMe}_{2}$ ), 26.65 (1C, 1`-C), 25.09 (1C, 6C), 22.04 (1C, 8-C), 21.55 (1C, 5-C), 20.39 (1C, 9-C). MS (EI) m/z (rel. int.): 542 (M $\left.\mathbf{M}^{+}, 29\right), 434$ (100), 389 (55). Anal. calcd. for $\mathrm{C}_{28} \mathrm{H}_{42} \mathrm{FeN}_{2} \mathrm{O}_{3} \mathrm{~S}$ (542.55): C, 61.98; H, 7.80; Fe, 10.29; N, 5.16; S, 5.91. Found: C, 62.07; H, 7.91; Fe, 10.48; N, 5.22; S $5.75 \%$.

Data of 36 (data for a diastereomeric mixture of two isomers in ratio 1:1): ${ }^{1} \mathrm{H} \mathrm{NMR}\left(250 \mathrm{MHz}, \mathrm{CDCl}_{3}, 300 \mathrm{~K}\right): \delta=4.16$ 4.11 (m, Fc), 4.12 (s, Cp), 4.10 (s, Cp), 4.10 (s, Cp), 4.09-4.00 (m, Fc), 3.96-3.92 (m, Fc), 3.32 (d, J = 14.5 Hz), 3.29 (d, $J=14.5 \mathrm{~Hz}), 2.89\left(\mathrm{~s}, \mathrm{SO}_{2} \mathrm{NMe}_{2}\right), 2.88\left(\mathrm{~s}, \mathrm{SO}_{2} \mathrm{NMe}_{2}\right), 2.80-2.76(\mathrm{~m}), 2.70(\mathrm{~d}, J=14.6 \mathrm{~Hz}), 2.69(\mathrm{~d}, J=14.6 \mathrm{~Hz}), 2.67-$ $2.53(\mathrm{~m}), 2.49-2.35(\mathrm{~m}), 2.32-2.27(\mathrm{~m}), 2.24\left(\mathrm{~s}, \mathrm{NMe}_{2}\right), 2.23\left(\mathrm{~s}, \mathrm{NMe}_{2}\right), 2.16-2.81(\mathrm{~m}), 1.74-1.49(\mathrm{~m}), 1.46-1.22(\mathrm{~m})$, 1.09 (s, Me), 1.01 (s, Me), 1.89 (m, Me), 1.85 (s, Me). ${ }^{13} \mathrm{C} \mathrm{NMR}\left(62.90 \mathrm{MHz}, \mathrm{CDCl}_{3}, 300 \mathrm{~K}\right): \delta=216.08(1 \mathrm{C}, \mathrm{C}=\mathrm{O})$, 215.85 (1C, C=O), 94.75 (1C, quaternary Fc), 93.80 (1C, quaternary $\mathrm{Fc}$ ), 69.03 (1C, Fc), 68.54 (5C, Cp), 68.45 (5C, $\mathrm{Cp}), 68.40$ (1C, Fc), 67.72 (1C, Fc), 67.68 (1C, Fc), 67.27 (1C, Fc), 66.82 (1C, Fc), 66.45 (1C, Fc), $66.22(1 \mathrm{C}, \mathrm{Fc})$, $60.29\left(1 \mathrm{C}, \mathrm{CH}_{2}\right), 59.93\left(1 \mathrm{C}, \mathrm{CH}_{2}\right), 59.70(1 \mathrm{C}$, quaternary), $58.52(1 \mathrm{C}$, quaternary), $57.94(1 \mathrm{C}, \mathrm{CH}), 53.85(1 \mathrm{C}, \mathrm{CH})$, 47.31 (1C, quaternary), 46.60 (2C, quaternary, $\mathrm{CH}), 46.60(1 \mathrm{C}, \mathrm{CH}), 45.57\left(2 \mathrm{C}, \mathrm{NMe}_{2}\right), 45.54\left(2 \mathrm{C}, \mathrm{NMe}_{2}\right), 44.04(1 \mathrm{C}$, 
$\left.\mathrm{CH}_{2}\right), 43.84\left(1 \mathrm{C}, \mathrm{CH}_{2}\right), 40.51(1 \mathrm{C}, \mathrm{CH}), 37.48\left(2 \mathrm{C}, \mathrm{SO}_{2} \mathrm{NMe}_{2}\right), 37.46\left(2 \mathrm{C}, \mathrm{SO}_{2} \mathrm{NMe}_{2}\right), 36.79(1 \mathrm{C}, \mathrm{CH}), 33.10(1 \mathrm{C}$, $\left.\mathrm{CH}_{2}\right), 30.86\left(1 \mathrm{C}, \mathrm{CH}_{2}\right), 29.35\left(1 \mathrm{C}, \mathrm{CH}_{2}\right), 26.23\left(1 \mathrm{C}, \mathrm{CH}_{2}\right), 25.38\left(1 \mathrm{C}, \mathrm{CH}_{2}\right), 25.26\left(1 \mathrm{C}, \mathrm{CH}_{2}\right), 25.01\left(1 \mathrm{C}, \mathrm{CH}_{2}\right), 21.68$ (1C, Me), 21.32 (1C, Me), $20.73\left(1 \mathrm{C}, \mathrm{CH}_{2}\right), 19.86$ (1C, Me), 19.55 (1C, Me).

4.10.

1-((1S,2R,4S,E)-3-(Ferrocenylmethylidene)-2-hydroxy-7,7-dimethyl-2-((6-methylpyridin-2yl)methyl)bicyclo[2.2.1]heptan-1-yl)- $N, N$-dimethylmethanesulfonamide 30

To a solution of 2,6-lutidine $(0.54 \mathrm{ml}, 4.62 \mathrm{mmol})$ in $12 \mathrm{ml}$ of THF was added dropwise $n$-BuLi (2.03 ml, 5.08 $\mathrm{mmol}$ of $2.5 \mathrm{M}$ solution in hexane) at $-60^{\circ} \mathrm{C}$. The mixture was stirred for $1 \mathrm{~h}$ at $-40^{\circ} \mathrm{C}$ and after cooling to $-60^{\circ} \mathrm{C}, 9(0.70$ $\mathrm{g}, 1.54 \mathrm{mmol}$ ) was added. The reaction progress was monitored by TLC (hexane/ethyl acetate $=2: 1$ ). After stirring for 1 $\mathrm{h}$ the mixture was quenched with water $(20 \mathrm{ml})$, extracted with $\mathrm{Et}_{2} \mathrm{O}(3 \times 30 \mathrm{ml})$ and the organic phase was dried over anhydrous $\mathrm{Na}_{2} \mathrm{SO}_{4}$. The solvent was evaporated in vacuo and the crude product was chromatographed (160 g silica gel, hexane/ethyl acetate $=5: 1)$ to give pure $30(0.450 \mathrm{~g}, 52 \%)$ as a deep orange solid and $\mathbf{3 7}(0.187 \mathrm{~g}, 22 \%)$ as an orange oil in form of diastereomeric mixture of two isomers (major 37a and minor $\mathbf{3 7 b}$ ) in ratio 10:1.

Data of 30: Mp $162-164^{\circ} \mathrm{C} .[\alpha]_{\mathrm{D}}^{20}=0$ (the measurement failed because no light penetrates through the deep colored solution even at $\left.c 0.02, \mathrm{CHCl}_{3}\right) .{ }^{1} \mathrm{H} \mathrm{NMR}\left(600 \mathrm{MHz}, \mathrm{CDCl}_{3}, 293 \mathrm{~K}\right): \delta=8.16(\mathrm{br} \mathrm{s}, 1 \mathrm{H}, \mathrm{OH}), 7.59\left(\mathrm{t}, 1 \mathrm{H}, 4^{`}-\mathrm{H}, J=7.5\right.$ $\mathrm{Hz}), 7.10\left(\mathrm{~d}, 1 \mathrm{H}, 5^{`}-\mathrm{H}, J=7.7 \mathrm{~Hz}\right), 7.06$ (d, 1H, $\left.3^{`}-\mathrm{H}, J=7.5 \mathrm{~Hz}\right), 5.50$ (s, 1H, 11-H), 4.18 (br s 1H, 16-H), 4.14* (br s $1 \mathrm{H}, 14-\mathrm{H}), 4.12$ (br s $1 \mathrm{H}, 13-\mathrm{H}), 4.11^{*}$ (br s, 1H, 15-H), 3.99 (s, 5H, Cp), 3.49 (d, 1H, 1 - $\left.\mathrm{H}_{\mathrm{b}}, J=14.7 \mathrm{~Hz}\right), 3.31(\mathrm{~d}, 1 \mathrm{H}$, $\left.10-\mathrm{H}_{\mathrm{a}}, J=14.6 \mathrm{~Hz}\right), 2.93\left(\mathrm{~d}, 1 \mathrm{H}, 1^{`}-\mathrm{H}_{\mathrm{a}}, J=14.7 \mathrm{~Hz}\right), 2.78\left(\mathrm{~s}, 6 \mathrm{H}, \mathrm{SO}_{2} \mathrm{NMe}_{2}\right), 2.77\left(\mathrm{~d}, 1 \mathrm{H}, 10-\mathrm{H}_{\mathrm{b}}, J=14.6 \mathrm{~Hz}\right), 2.73(\mathrm{~d}$, $1 \mathrm{H}, 4-\mathrm{H}, J=5.4 \mathrm{~Hz}), 2.59$ (s, 3H, 7`-H), 2.39-2.34 (m, 1H, 6- $\left.\mathrm{H}_{\text {exo }}\right), 2.09-2.00\left(\mathrm{~m}, 2 \mathrm{H}, 5-\mathrm{H}_{\text {exo }}, 6-\mathrm{H}_{\text {endo }}\right), 1.44-1.39(\mathrm{~m}, 1 \mathrm{H}$, 5- $\mathrm{H}_{\text {endo }}$ ), 1.05 (s, 3H, 8-H), 1.04 (s, 3H, 9-H). ${ }^{13} \mathrm{C} \mathrm{NMR}\left(150.92 \mathrm{MHz}, \mathrm{CDCl}_{3}, 293 \mathrm{~K}\right): \delta=159.18$ (1C, $\left.2^{\prime}-\mathrm{C}\right), 156.52$ (1C, 6 -C), 151.33 (1C, 3-C), 137.55 (1C, 4`-C), 122.50 (1C, 3`-C), 121.24 (1C, 5-C), 117.82 (1C, 11-C), 83.05 (1C, 12-C), 81.67 (1C, 2-C), 69.05 (1C, 13-C), 68.82 (5C, Cp), 68.22* (1C, 15-C), 68.14* (1C, 14-C), 67.91 (1C, 16-C), 53.62 (1C, 1-C), 50.33 (1C, 4-C), 50.25 (1C, 7-C), 43.59 (1C, 10-C), 43.43 (1C, 1 -C), 37.64 (2C, $\left.\mathrm{SO}_{2} \mathrm{NMe}_{2}\right), 26.09$ (1C, 6-C), 25.17 (1C, 5-C), 24.27 (1C, 7`-C), 22.00 (1C, 8-C), 20.95 (1C, 9-C). MS (EI) m/z (rel. int.): 562 (M $\left.{ }^{+}, 35\right)$, 455 (100), 347 (33), 281 (26), 107 (40). Anal. calcd. for $\mathrm{C}_{30} \mathrm{H}_{38} \mathrm{FeN}_{2} \mathrm{O}_{3} \mathrm{~S}$ (562.54): C, 64.05; H, 6.81; Fe, 9.93; N, 4.98; S, 5.70. Found: C, 64.20; H, 6.76; Fe, 9.77; N, 5.03; S, $5.80 \%$.

Data for 37a: (the major diastereoisomer 37a was isolated in $43 \mathrm{mg}$ quantity as orange-yellow solid by crystallization of mixture 37a/37b from heptane/ $\left.\mathrm{CH}_{2} \mathrm{Cl}_{2}=90: 10\right) .{ }^{1} \mathrm{H}$ NMR $\left(250 \mathrm{MHz}, \mathrm{CDCl}_{3}, 300 \mathrm{~K}\right): \delta=7.51\left(\mathrm{t}, 1 \mathrm{H}, 4^{\prime}-\mathrm{H}, J=7.6 \mathrm{~Hz}\right)$, $7.06(\mathrm{~d}, 1 \mathrm{H}, 5-\mathrm{H}, J=7.6 \mathrm{~Hz}), 7.00\left(\mathrm{~d}, 1 \mathrm{H}, 3^{`}-\mathrm{H}, J=7.8 \mathrm{~Hz}\right), 4.13-4.08(\mathrm{~m}, 4 \mathrm{H}, \mathrm{Fc}), 4.10$ (s, 5H, Cp), 3.68-3.61 (m, 1H, $\left.1^{`}-\mathrm{H}_{\mathrm{a}}\right), 3.33-3.26(\mathrm{~m}, 1 \mathrm{H}, 4-\mathrm{H}), 3.19\left(\mathrm{~d}, 1 \mathrm{H}, 10-\mathrm{H}_{\mathrm{a}}, J=14.7 \mathrm{~Hz}\right), 3.07\left(\mathrm{dd}, 1 \mathrm{H}, 1^{`}-\mathrm{H}_{\mathrm{b}}, J=13.7,5.5 \mathrm{~Hz}\right), 2.80(\mathrm{~s}, 6 \mathrm{H}$, $\left.\mathrm{SO}_{2} \mathrm{NMe}_{2}\right), 2.63-2,58\left(\mathrm{~m}, 2 \mathrm{H}, 10-\mathrm{H}_{\mathrm{b}}, 11\right), 2.55\left(\mathrm{~s}, 3 \mathrm{H}, 7^{-}-\mathrm{H}\right), 2.43-2.31^{*}\left(\mathrm{~m}, 1 \mathrm{H}, 5-\mathrm{H}_{\text {exo }}\right), 1.67-1.64(\mathrm{~m}, 1 \mathrm{H}, 3-\mathrm{H}), 1.59-$ $1.53\left(\mathrm{~m}, 2 \mathrm{H}, 6-\mathrm{H}_{\text {endo }}, 6-\mathrm{H}_{\text {exo }}\right), 1.42-1.31^{*}\left(\mathrm{~m}, 1 \mathrm{H}, 5-\mathrm{H}_{\text {endo }}\right), 1.06^{*}(\mathrm{~s}, 3 \mathrm{H}, 8-\mathrm{H}), 0.77^{*}(\mathrm{~s}, 3 \mathrm{H}, 9-\mathrm{H}) .{ }^{13} \mathrm{C} \mathrm{NMR}(62.90 \mathrm{MHz}$, $\left.\mathrm{CDCl}_{3}, 300 \mathrm{~K}\right): \delta=214.65$ (1C, 2-C), 160.05 (1C, $\left.2{ }^{`}-\mathrm{C}\right), 157.75$ (1C, 6`C), 136.38 (1C, $\left.4^{`}-\mathrm{C}\right), 120.64$ (1C, $\left.3^{`}-\mathrm{C}\right)$, 120.36 (1C, 5-C), 92.69 (1C, 12-C), 69.64 (1C, Fc), 68.50 (5C, Cp), 67.22 (1C, Fc), 67.04 (1C, Fc), $66.27(1 \mathrm{C}, \mathrm{Fc})$, 59.18 (1C, 1-C), 55.33 (1C, 11-C), 47.62 (1C, 3-C), 46.65 (1C, 7-C), 44.74 (1C, 1-C), 43.92 (1C, 10-C), 37.39 (2C, $\mathrm{SO}_{2} \mathrm{NMe}_{2}$ ), 37.35 (1C, 4-C), 25.01* (1C, 5-C), 24.50 (1C, 7-C), 20.82* (1C, 6-C), $20.06^{*}$ (1C, 8-C), 19.36* (1C, 9-C).

4.11.

1-((1S,2R,4S,E)-3-Ferrocenylmethylidene-2-hydroxy-2-(1-isopropyl-1H-imidazol-2-yl)-7,7dimethylbicyclo[2.2.1] heptan-1-yl)- $N, N$-dimethylmathanesulfonamide 31

To a solution of 1-isopropyl- $1 H$-imidazole $(0.22 \mathrm{~g}, 1.98 \mathrm{mmol})$ in $4 \mathrm{ml}$ of hexane and $4 \mathrm{ml}$ of THF was added dropwise $n$ - $\mathrm{BuLi}\left(1.20 \mathrm{ml}, 2.97 \mathrm{mmol}\right.$ of $2.5 \mathrm{M}$ solution in hexane) at $-90^{\circ} \mathrm{C}$. The mixture was stirring for $1 \mathrm{~h}$ at this temperature, after which $9(0.30 \mathrm{~g}, 0.66 \mathrm{mmol})$ was added. The reaction progress was monitored by $\mathrm{TLC}\left(\mathrm{Et}_{2} \mathrm{O} / \mathrm{NH}_{3}=\right.$ 6:0.01). After stirring for additional $1.5 \mathrm{~h}$ at $-40^{\circ} \mathrm{C}$, the mixture was quenched with water $(5 \mathrm{ml})$, extracted with $\mathrm{Et}_{2} \mathrm{O}$ $(3 \times 20 \mathrm{ml})$ and the organic phase was dried over anhydrous $\mathrm{Na}_{2} \mathrm{SO}_{4}$. The solvent was evaporated in vacuo and the crude product was chromatographed (90 g silica gel, $\left.\mathrm{Et}_{2} \mathrm{O} / \mathrm{NH}_{3}=6: 0.01\right)$, to give $31(0.178 \mathrm{~g}, 48 \%$ ) as a yellow solid. Mp 86$87^{\circ} \mathrm{C} .[\alpha]_{\mathrm{D}}^{20}=-339.0\left(c 0.64, \mathrm{CHCl}_{3}\right) .{ }^{1} \mathrm{H} \mathrm{NMR}\left(250 \mathrm{MHz}, \mathrm{CDCl}_{3}, 300 \mathrm{~K}\right): \delta=7.01\left(\mathrm{~d}, 1 \mathrm{H}, 2^{\prime}-\mathrm{H}, J=1.3 \mathrm{~Hz}\right), 6.95(\mathrm{~d}$, $1 \mathrm{H}, 3-\mathrm{H}, J=1.3 \mathrm{~Hz}$ ), 6.33 (br s, $1 \mathrm{H}, \mathrm{OH}), 4.76$ (quint, $1 \mathrm{H}, 4-\mathrm{H}), 4.48-4.47$ (m, 1H, 16-H), 4.28-4.26* (m, 1H, 14-H), $4.22-4.20^{*}(\mathrm{~m}, 1 \mathrm{H}, 15-\mathrm{H}), 4.18-4.16(\mathrm{~m}, 1 \mathrm{H}, 13-\mathrm{H}), 4.16(\mathrm{~s}, 5 \mathrm{H}, \mathrm{Cp}), 3.79\left(\mathrm{~d}, 1 \mathrm{H}, 10-\mathrm{H}_{\mathrm{a}}, J=14.9 \mathrm{~Hz}\right), 3.11(\mathrm{~d}, 1 \mathrm{H}, 10-$ $\left.\mathrm{H}_{\mathrm{b}}, J=14.9 \mathrm{~Hz}\right), 3.06(\mathrm{~d}, 1 \mathrm{H}, 4-\mathrm{H}, J=4.3 \mathrm{~Hz}), 2.85\left(\mathrm{~s}, 6 \mathrm{H}, \mathrm{SO}_{2} \mathrm{NMe}_{2}\right), 2.42\left(\mathrm{ddd}, 1 \mathrm{H}, 6-\mathrm{H}_{\text {exo }}, J=13.8,12.2,3.6 \mathrm{~Hz}\right)$, 2.09-1.95 (m, 1H, 5- $\left.\mathrm{H}_{\text {exo }}\right), 1.57-1.42\left(\mathrm{~m}, 1 \mathrm{H}, 5-\mathrm{H}_{\text {endo }}\right), 1.39$ (d, 3H, 5- $\left.\mathrm{H}_{\mathrm{a}}, J=6.8 \mathrm{~Hz}\right), 1.32(\mathrm{~s}, 3 \mathrm{H}, 8-\mathrm{H}), 1.22(\mathrm{~s}, 3 \mathrm{H}, 9-$ $\mathrm{H}), 1.20\left(\mathrm{~d}, 3 \mathrm{H}, 5-\mathrm{H}_{\mathrm{b}}, J=6.8 \mathrm{~Hz}\right), 1.18-1.05\left(\mathrm{~m}, 1 \mathrm{H}, 6-\mathrm{H}_{\text {endo }}\right) .{ }^{13} \mathrm{C} \mathrm{NMR}\left(62.90 \mathrm{MHz}, \mathrm{CDCl}_{3}, 300 \mathrm{~K}\right): \delta=149.31(1 \mathrm{C}$, $1 `-\mathrm{C}), 144.32$ (1C, 3-C), 126.74 (1C, 2`C), 124.60 (1C, 11-C), 117.02 (1C, 3`-C), 83.30 (1C, 12-C), 81.15 (1C, 2-C), 70.23 (1C, 13-C), 69.02* (1C, 14-C), 69.00 (5C, Cp), 68.28* (1C, 15-C), 67.58 (1C, 16-C), 55.38 (1C, 1-C), 51.14 (1C, 4-C), 49.44 (1C, 7-C), 47.84 (1C, 4-C), 42.79 (1C, 10-C), 37.67 (2C, $\mathrm{SO}_{2} \mathrm{NMe}_{2}$ ), 25.62 (1C, 6-C), 24.32 (1C, 5-C),

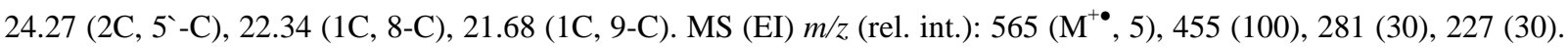


Anal. calcd. for $\mathrm{C}_{29} \mathrm{H}_{39} \mathrm{FeN}_{3} \mathrm{O}_{3} \mathrm{~S}$ (565.55): C, 61.59; H, 6.95; Fe, 9.87; N, 7.43; S, 5.67. Found: C, 61.70; H, 6.88; Fe, 9.94; N, 7.57; S, $5.59 \%$.

4.12.

1-((1S,2R,4S,E)-2-(Benzo[b]thiophen-2-yl)-3-ferrocenylmethylidene-2-hydroxy-7,7dimethylbicyclo[2.2.1]heptan-1-yl)- $N, N$-dimethylmethanesulfonamide 32

To a solution of benzothiophene $(0.27 \mathrm{~g}, 1.98 \mathrm{mmol})$ in $20 \mathrm{ml}$ of THF was added dropwise $n$-BuLi $(1.36 \mathrm{ml}$, $2.18 \mathrm{mmol}$ of $1.6 \mathrm{M}$ solution in hexane) at $-50^{\circ} \mathrm{C}$. The mixture was stirred for $30 \mathrm{~min}$. at $-10^{\circ} \mathrm{C}$, then cooled to $-60^{\circ} \mathrm{C}$ and $9(0.30 \mathrm{~g}, 0.66 \mathrm{mmol})$ was added at this temperature. After slowly warming to room temperature and stirring for 40 min, the mixture was quenched with water $(5 \mathrm{ml})$, extracted with $\mathrm{Et}_{2} \mathrm{O}(3 \times 20 \mathrm{ml})$ and the organic phase was dried over anhydrous $\mathrm{Na}_{2} \mathrm{SO}_{4}$. The solvent was evaporated in vacuo and the crude product was chromatographed (180 g silica gel, toluene $\left./ \mathrm{Et}_{2} \mathrm{O}=20: 1\right)$, to give $32(0.263 \mathrm{~g}, 65 \%)$ as a yellow solid. $\mathrm{Mp} 113-115^{\circ} \mathrm{C}$. $[\alpha]_{\mathrm{D}}^{20}=-256.3\left(c 1.00, \mathrm{CHCl}_{3}\right) .{ }^{1} \mathrm{H}$ NMR (600 MHz, $\left.\mathrm{CDCl}_{3}, 293 \mathrm{~K}\right): \delta=7.72^{*}(\mathrm{dd}, 1 \mathrm{H}, 7 `-\mathrm{H}, J=6.1,2.8 \mathrm{~Hz}), 7.33^{*}(\mathrm{dd}, 1 \mathrm{H}, 4-\mathrm{H}, J=6.1,2.8 \mathrm{~Hz}), 7.35-$ 7.32 (m, 2H, $5-\mathrm{H}, 6-\mathrm{H}), 7.23(\mathrm{~s}, 1 \mathrm{H}, 2-\mathrm{H}), 6.27(\mathrm{~s}, 1 \mathrm{H}, 11-\mathrm{H}), 4.44-4.43(\mathrm{~m}, 1 \mathrm{H}, 16-\mathrm{H}), 4.33-4.32^{*}(\mathrm{~m}, 1 \mathrm{H}, 14-\mathrm{H})$, 4.31-4.30 (m, 1H, 13-H), 4.27-4.26* (m, 1H, 15-H), $4.21(\mathrm{~s}, 5 \mathrm{H}, \mathrm{Cp}), 3.51\left(\mathrm{~d}, 1 \mathrm{H}, 10-\mathrm{H}_{\mathrm{a}}, J=14.9 \mathrm{~Hz}\right), 3.34(\mathrm{~d}, 1 \mathrm{H}, 10-$ $\left.\mathrm{H}_{\mathrm{b}}, J=14.9 \mathrm{~Hz}\right), 2.94(\mathrm{~d}, 1 \mathrm{H}, 4-\mathrm{H}, J=4.6), 2.92\left(\mathrm{~s}, 6 \mathrm{H}, \mathrm{SO}_{2} \mathrm{NMe}_{2}\right), 2.75(\mathrm{~s}, 1 \mathrm{H}, \mathrm{OH}), 2.35\left(\mathrm{ddd}, 1 \mathrm{H}, 6-\mathrm{H}_{e x o}, J=13.2\right.$, $12.3,4.6 \mathrm{~Hz}), 2.13-2.07\left(\mathrm{~m}, 1 \mathrm{H}, 5-\mathrm{H}_{\text {exo }}\right), 1.59-1.55\left(\mathrm{~m}, 1 \mathrm{H}, 5-\mathrm{H}_{\text {endo }}\right), 1.51-1.47\left(\mathrm{~m}, 1 \mathrm{H}, 6-\mathrm{H}_{\text {endo }}\right), 1.27(\mathrm{~s}, 3 \mathrm{H}, 8-\mathrm{H}), 1.26$ (s, 3H, 9-H). ${ }^{13} \mathrm{C}$ NMR (150.92 MHz, $\left.\mathrm{CDCl}_{3}, 293 \mathrm{~K}\right): \delta=150.15$ (1C, 1`-C), 144.32 (1C, 3-C), 139.77 (1C, $\left.8^{`}-\mathrm{C}\right)$, 138.90 (1C, $\left.3^{`}-\mathrm{C}\right), 124.40$ (2C, $\left.5^{`}-\mathrm{C}, 6^{`}-\mathrm{C}\right), 123.56$ (1C, $\left.4^{`}-\mathrm{C}\right), 123.29$ (1C, $\left.2^{`}-\mathrm{C}\right), 122.73$ (1C, 11-C), 122.16 (1C, $\left.7^{`}-\mathrm{C}\right)$, 85.35 (1C, 12-C), 81.15 (1C, 2-C), 69.45* (1C, 15-C), 68.90* (1C, 14-C), 68.88 (1C, 13-C), 68.82 (5C, Cp), 67.97 (1C, 16-C), 55.02 (1C, 1-C), 51.47 (1C, 4-C), 49.98 (1C, 7-C), 43.49 (1C, 10-C), $37.76\left(2 \mathrm{C}, \mathrm{SO}_{2} \mathrm{NMe}_{2}\right) 27.59(1 \mathrm{C}, 6-\mathrm{C})$, 22.44 (1C, 5-C), 22.43 (1C, 8-C), 21.89 (1C, 9-C). MS (EI) m/z (rel. int.): 589 (M $\left.\mathbf{M}^{+}, 100\right), 573$ (27), 480 (77), 463 (19), 415 (51), 343 (74). Anal. calcd. for $\mathrm{C}_{31} \mathrm{H}_{35} \mathrm{FeNO}_{3} \mathrm{~S}_{2}$ (589.59): C, 63.15; H, 5.98; Fe, 9.47; N, 2.38; S, 10.88. Found: C, $63.31 ; \mathrm{H} 5.88 ; \mathrm{Fe}, 9.54 ; \mathrm{N}, 2.26 ; \mathrm{S}, 10.69 \%$.

\subsection{1-((1S,2R,4S,E)-3-Ferrocenylmethylidene-2-(dibenzo $[b, d]$ thiophen-4-yl)-2-hydroxy-7,7- dimethylbicyclo[2.2.1] heptan-1-yl)- $N, N$-dimethylmethanesulfonamide 33}

To a solution of dibenzothiophene $(0.37 \mathrm{~g}, 1.98 \mathrm{mmol})$ in $20 \mathrm{ml}$ of THF was added dropwise $n$-BuLi $(1.36 \mathrm{ml}$, $2.18 \mathrm{mmol}$ of $1.6 \mathrm{M}$ solution in hexane) at $-20^{\circ} \mathrm{C}$. The mixture was stirred for $3 \mathrm{~h}$ at $0^{\circ} \mathrm{C}$ and $2 \mathrm{~h}$ at room temperature. Next, $9(0.30 \mathrm{~g}, 0.66 \mathrm{mmol})$ was added at $0^{\circ} \mathrm{C}$. After stirring for $1 \mathrm{~h}$ at room temperature the mixture was quenched with water $(5 \mathrm{ml})$, extracted with $\mathrm{Et}_{2} \mathrm{O}(3 \times 20 \mathrm{ml})$ and the organic phase was dried over anhydrous $\mathrm{Na}_{2} \mathrm{SO}_{4}$. The solvent was evaporated in vacuo and the crude product was chromatographed (40 g silica gel, toluene/ $\left.\mathrm{Et}_{2} \mathrm{O}=20: 1\right)$, to give pure 33 $(0.111 \mathrm{~g}, 25 \%)$ as orange solid and $\mathbf{3 8}(0.186 \mathrm{~g}, 44 \%)$ as a diastereomeric mixture of two isomers (major 38a and minor $\mathbf{3 8 b}$ ) in ratio 10:1 as orange-yellow solid.

Data of 33: Mp 158- $160^{\circ} \mathrm{C}$ with decomp. $[\alpha]_{\mathrm{D}}^{20}=-216.2\left(c 0.63, \mathrm{CHCl}_{3}\right) .{ }^{1} \mathrm{H} \mathrm{NMR}\left(600 \mathrm{MHz}, \mathrm{CDCl}_{3}, 293 \mathrm{~K}\right): \delta=8.17-$ 8.15 (m, 2H, 4`-H, 7`-H), 7.83-7.80 (m, 1H, 10`-H), 7.46-7.43 (m, 3H, 3`-H, 8`-H, 9`-H), 7.38-7.37 (br d, 1H, $2 `-\mathrm{H})$, 6.13 (s, 1H, 11-H), 4.45 (br s, 1H, 16-H), 4.30* (br s, 1H, 14-H), 4.27 (br s, 1H, 13-H), 4.27* (br s, 1H, 15-H), 4.17 (s, $5 \mathrm{H}, \mathrm{Cp}), 3.60\left(\mathrm{~d}, 1 \mathrm{H}, 10-\mathrm{H}_{\mathrm{a}}, J=14.8 \mathrm{~Hz}\right), 3.42\left(\mathrm{~d}, 1 \mathrm{H}, 10-\mathrm{H}_{\mathrm{b}}, J=14.8 \mathrm{~Hz}\right), 3.00(\mathrm{~d}, 1 \mathrm{H}, 4-\mathrm{H}, J=4.4 \mathrm{~Hz}), 2.65(\mathrm{~s}, 6 \mathrm{H}$, $\left.\mathrm{SO}_{2} \mathrm{NMe}_{2}\right), 2.45-2.43\left(\mathrm{~m}, 2 \mathrm{H}, 6-\mathrm{H}_{\text {exo }}, \mathrm{OH}\right), 2.20-2.14\left(\mathrm{~m}, 1 \mathrm{H}, 5-\mathrm{H}_{\text {exo }}\right), 1.72-1.67\left(\mathrm{~m}, 1 \mathrm{H}, 5-\mathrm{H}_{\text {endo }}\right), 1.55-1.50(\mathrm{~m}, 1 \mathrm{H}, 6-$ $\mathrm{H}_{\text {endo }}$ ), 1.35 (s, 3H, 8-H), 1.26 (s, 3H, 9-H). $\left.{ }^{13} \mathrm{C} \mathrm{NMR} \mathrm{(150.92} \mathrm{MHz,} \mathrm{CDCl}_{3}, 293 \mathrm{~K}\right): \delta=140.28$ (1C, 3-C), 140.28 (1C, $\left.11^{`}-\mathrm{C}\right), 138.71$ (1C, $\left.12^{`}-\mathrm{C}\right), 138.54$ (1C, $\left.1^{`}-\mathrm{C}\right), 128.40$ (1C, $\left.2^{`}-\mathrm{C}\right), 137.11$ (1C, $\left.5^{`}-\mathrm{C}\right), 134.77$ (1C, $\left.6^{`}-\mathrm{C}\right), 126.80$ (1C, $9^{`}$ C), 124.21 (1C, 8`-C), 123.17 (1C, 3`-C), 122.86 (1C, 11-C), 121.60 (1C, 10`-C), 121.46 (1C, 7`-C), 121.32 (1C, $\left.4{ }^{`}-\mathrm{C}\right)$, 88.69 (1C, 2-C), 81.93 (1C, 12-C), 69.60 (1C, 13-C), 68.97* (1C, 15-C), 68.89* (1C, 14-C), 68.72 (5C, Cp), 67.69 (1C, 16-C), 57.09 (1C, 1-C), 51.96 (1C, 4-C), 50.68 (1C, 7-C), 44.95 (1C, 10-C), 37.63 (2C, $\left.\mathrm{SO}_{2} \mathrm{NMe}_{2}\right), 25.88$ (1C, 6-C), 24.42 (1C, 5-C), 22.96 (1C, 8-C), 22.01 (1C, 9-C). MS (EI) m/z (rel. int.): 639 (M $\left.\mathbf{M}^{+}, 100\right), 530$ (100), 465 (53), 393 (20). Anal. calcd. for $\mathrm{C}_{35} \mathrm{H}_{37} \mathrm{FeNO}_{3} \mathrm{~S}_{2}$ (639.65): C, 65.72; H, 5.83; Fe, 8.73; N, 2.19; S, 10.03. Found: C, 65.62; H 5.69; Fe, $8.83 ; \mathrm{N}, 2.08 ; \mathrm{S}, 10.17 \%$.

Data for 38a: (the major diastereoisomer 38a was isolated in $36 \mathrm{mg}$ quantity as orange-yellow solid by crystallization of mixture 38a/38b from heptane $\left./ \mathrm{CH}_{2} \mathrm{Cl}_{2}=90: 10\right)$. ${ }^{1} \mathrm{H} \mathrm{NMR}\left(250 \mathrm{MHz}, \mathrm{CDCl}_{3}, 300 \mathrm{~K}\right): \delta=8.19-8.09(\mathrm{~m}, 2 \mathrm{H}), 7.90-7.86$ $(\mathrm{m}, 1 \mathrm{H}), 7.60-7.44(\mathrm{~m}, 4 \mathrm{H}), 4.17-4.14(\mathrm{~m}, 4 \mathrm{H}, \mathrm{Fc}), 3.96$ (d, 1H, J = 11.4 Hz), 3.57 (s, 5H, Cp), $3.19(\mathrm{~d}, 1 \mathrm{H}, J=14.5$ $\mathrm{Hz}), 2.76\left(\mathrm{~s}, 6 \mathrm{H}, \mathrm{SO}_{2} \mathrm{NMe}_{2}\right), 2.70-2.61(\mathrm{~m}, 2 \mathrm{H}), 2.53-2.44(\mathrm{~m}, 1 \mathrm{H}), 2.09-2.01(\mathrm{~m}, 1 \mathrm{H}), 1.67-1.60(\mathrm{~m}, 1 \mathrm{H}), 1.44-1.25(\mathrm{~m}$, $2 \mathrm{H}), 1.21$ (s, 3H), 1.11 (s, 3H). ${ }^{13} \mathrm{C} \mathrm{NMR}\left(62.90 \mathrm{MHz}, \mathrm{CDCl}_{3}, 300 \mathrm{~K}\right): \delta=213.21(1 \mathrm{C}, \mathrm{C}=\mathrm{O}), 139.96$ (1C, quaternary), 139.87 (1C, quaternary), 138.73 (1C, quaternary), 136.76 (1C, quaternary), 135.30 (1C, quaternary), $126.57(1 \mathrm{C}, \mathrm{CH}$ $\mathrm{Ar}), 124.78$ (1C, CH Ar), 124.36 (1C, CH Ar), 124.29 (1C, CH Ar), 122.66 (1C, CH Ar), 121.84 (1C, CH Ar), 119.62 (1C, CH Ar), 91.95 (1C, quaternary Fc), 70.26 (1C, CH Fc), 68.60 (1C, CH Fc), 68.52 (5C, Cp), 66.25 (1C, CH Fc), $65.70(1 \mathrm{C}, \mathrm{CH} \mathrm{Fc}), 60.42(1 \mathrm{C}, \mathrm{CH}), 58.54$ (1C, quaternary), 47.27 (1C, quaternary), $46.69(1 \mathrm{C}, \mathrm{CH}), 46.39(1 \mathrm{C}, \mathrm{CH})$, $43.06\left(1 \mathrm{C}, \mathrm{CH}_{2}\right), 37.44$ (2C, Me), $29.44\left(1 \mathrm{C}, \mathrm{CH}_{2}\right), 24.38$ (1C, $\left.\mathrm{CH}_{2}\right), 21.94$ (1C, Me), 21.53 (1C, Me). 
4.14. 1-((1S,2R,4S,E)-3-Ferrocenylmethylidene-2-hydroxy-7,7-dimethyl-2-(5-(trimethylsilyl)thiophen-2yl)bicyclo[2.2.1]heptan-1-yl)-N,N-dimethylmethanesulfonamide 34

To a solution of trimethy-2-thienylsilane $(0.31 \mathrm{~g}, 1.98 \mathrm{mmol})$ in $15 \mathrm{ml}$ of THF was added dropwise $n$-BuLi $\left(1.09 \mathrm{ml}, 2.73 \mathrm{mmol}\right.$ of $2.5 \mathrm{M}$ solution in hexane) at $0^{\circ} \mathrm{C}$. The mixture was stirred for $1 \mathrm{~h}$ at room temperature and then $9(0.30 \mathrm{~g}, 0.66 \mathrm{mmol})$ was added. The reaction progress was monitored by $\mathrm{TLC}$ (petroleum ether/ $\left.\operatorname{Et}_{2} \mathrm{O}=2: 1\right)$ until 9 was consumed. After stirring for $30 \mathrm{~min}$. at room temperature, the reaction was quenched with water $(5 \mathrm{ml})$, extracted with $\mathrm{Et}_{2} \mathrm{O}(3 \times 20 \mathrm{ml})$ and the organic phase was dried over anhydrous $\mathrm{Na}_{2} \mathrm{SO}_{4}$. The solvent was evaporated in vacuo and the crude product was chromatographed $\left(250 \mathrm{~g}\right.$ silica gel, petroleum ether/ $\left.\mathrm{Et}_{2} \mathrm{O}=5: 1\right)$, to give $34(0.197 \mathrm{~g}, 47 \%)$ as a yellow solid. $\mathrm{Mp} 88-90^{\circ} \mathrm{C}$. $[\alpha]_{\mathrm{D}}^{20}=-210.2\left(c 1.00, \mathrm{CHCl}_{3}\right) .{ }^{1} \mathrm{H} \mathrm{NMR}\left(250 \mathrm{MHz}, \mathrm{CDCl}_{3}, 300 \mathrm{~K}\right): \delta=7.14\left(\mathrm{~d}, 1 \mathrm{H}, 3^{\prime}-\mathrm{H}\right)$, 7.07 (s, 1H, 2-H), 6.25 (s, 1H, 11-H), 4.41-4.40 (m, 1H, 16-H), 4.29-4.27 (m, 2H, 14-H, 15-H), 4.25-4.23 (m, 1H, 13H), 4.19 (s, 5H, Cp), $3.49\left(\mathrm{~d}, 1 \mathrm{H}, 10-\mathrm{H}_{\mathrm{a}}, J=14.9 \mathrm{~Hz}\right), 3.25\left(\mathrm{~d}, 1 \mathrm{H}, 10-\mathrm{H}_{\mathrm{b}}, J=14.9 \mathrm{~Hz}\right), 2.90\left(\mathrm{~s}, 6 \mathrm{H}, \mathrm{SO}_{2} \mathrm{NMe}_{2}\right), 2.89(\mathrm{br}$ $\mathrm{d}, 1 \mathrm{H}, 4-\mathrm{H}), 2.57(\mathrm{~s}, 1 \mathrm{H}, \mathrm{OH}), 2.36-2.24\left(\mathrm{~m}, 1 \mathrm{H}, 6-\mathrm{H}_{\text {exo }}\right), 2.11-1.98\left(\mathrm{~m}, 1 \mathrm{H}, 5-\mathrm{H}_{\text {exo }}\right), 1.57-1.46\left(\mathrm{~m}, 1 \mathrm{H}, 5-\mathrm{H}_{\text {endo }}\right), 1.37-$ $1.31\left(\mathrm{~m}, 1 \mathrm{H}, 6-\mathrm{H}_{\text {endo }}\right), 1.21(\mathrm{~s}, 6 \mathrm{H}, 8-\mathrm{H}, 9-\mathrm{H}), 0.32\left(\mathrm{~s}, 9 \mathrm{H}, \mathrm{SiMe}_{3}\right) .{ }^{13} \mathrm{C} \mathrm{NMR}\left(62.90 \mathrm{MHz}, \mathrm{CDCl}_{3}, 300 \mathrm{~K}\right): \delta=154.77$ (1C, 4-C), 150.44 (1C, 1`C), 140.15 (1C, 3-C), 133.26 (1C, 3`C), 127.57 (1C, 2`C), 122.16 (1C, 11-C), 84.84 (1C, 12-C), 82.18 (1C, 2-C), 69.44* (1C, 15-C), 68.80 (5C, Cp), 68.76* (1C, 14-C), 68.69 (1C, 13-C), 67.76 (1C, 16-C), 55.09 (1C, 1-C), 51.24 (1C, 4-C), 49.72 (1C, 7-C), 43.24 (1C, 10-C), 37.66 (2C, $\mathrm{SO}_{2} \mathrm{NMe}_{2}$ ), 27.38 (1C, 6-C), 24.46 (1C, 5-C), 22.38 (1C, 8-C), 21.84 (1C, 9-C), -0.07 (3C, SiMe 3 ). MS (EI) m/z (rel. int.): 611 (M+•, 64), 503 (31), 437 (100). Anal. calcd. for $\mathrm{C}_{30} \mathrm{H}_{41} \mathrm{FeNO}_{3} \mathrm{~S} 2 \mathrm{Si}$ (611.71): C, 58.90; H, 6.76; Fe, 9.13; N, 2.29; S, 10.48. Found: C, 59.02; H, 6.58; Fe, 9.35; N, 2.39; S, $10.27 \%$.

4.15.

1-((1S,2R,4S,E)-2-(Benzo[d]thiazol-2-yl)-3-ferrocenylmethylidene-2-hydroxy-7,7dimethylbicyclo[2.2.1] heptan-1-yl)- $N, N$-dimethylmethanesulfonamide 35

To a solution of benzothiazole $(0.45 \mathrm{~g}, 3.3 \mathrm{mmol})$ in $10 \mathrm{ml}$ of THF was added dropwise $n$-BuLi (1.40 ml, 3.47 mmol of $2.5 \mathrm{M}$ solution in hexane) at $-90^{\circ} \mathrm{C}$. The mixture was stirredfor $1 \mathrm{~h}$ at $-90^{\circ} \mathrm{C}$ and then was added $9(0.50 \mathrm{~g}, 1.10$ $\mathrm{mmol}$ ) was added. After stirring for $40 \mathrm{~min}$. at $-40^{\circ} \mathrm{C}$, the mixture was quenched with water $(10 \mathrm{ml})$, extracted with $\mathrm{Et}_{2} \mathrm{O}$ $(3 \times 30 \mathrm{ml})$ and the organic phase was dried $\left(\mathrm{Na}_{2} \mathrm{SO}_{4}\right)$. The solvent was evaporated in vacuo and the crude product was chromatographed ( $170 \mathrm{~g}$ silica gel, hexane/acetone $=5: 1)$, to give $0.289 \mathrm{~g}$ of a mixture of $\mathbf{9}$ and $\mathbf{3 5}$. The crystallization from hexane/ $\mathrm{CH}_{2} \mathrm{Cl}_{2}=90: 10$ provided pure $35(0.167 \mathrm{~g}, 25 \%)$ as a yellow solid. Mp 156-158 ${ }^{\circ} \mathrm{C}$. $[\alpha]_{\mathrm{D}}^{20}=-208.9(c 0.34$, $\left.\mathrm{CHCl}_{3}\right) .{ }^{1} \mathrm{H}$ NMR $\left(600 \mathrm{MHz}, \mathrm{CDCl}_{3}, 293 \mathrm{~K}\right): \delta=7.98\left(\mathrm{~d}, 1 \mathrm{H}, 6^{`}-\mathrm{H}, J=8.0 \mathrm{~Hz}\right), 7.87\left(\mathrm{~d}, 1 \mathrm{H}, 33^{`} \mathrm{H}, J=8.0 \mathrm{~Hz}\right), 7.49(\mathrm{dt}$, $1 \mathrm{H}, 4-\mathrm{H}, J=7.2,0.9 \mathrm{~Hz}), 7.40$ (dt, 1H, 5 - H, $J=7.2,0.9 \mathrm{~Hz}), 6.31(\mathrm{~s}, 1 \mathrm{H}, 11-\mathrm{H}), 4.44$ (br s, 1H, 16-H), 4.32 (br s, 1H, $13-\mathrm{H}), 4.30^{*}$ (br s, $\left.1 \mathrm{H}, 14-\mathrm{H}\right), 4.28(\mathrm{~s}, 1 \mathrm{H}, \mathrm{OH}), 4.24^{*}$ (br s, 1H, 15-H), 4.22 (s, 5H, Cp), $3.80\left(\mathrm{~d}, 1 \mathrm{H}, 10-\mathrm{H}_{\mathrm{a}}, J=14.8\right.$ $\mathrm{Hz}), 3.25\left(\mathrm{~d}, 1 \mathrm{H}, 10-\mathrm{H}_{\mathrm{b}}, J=14.8 \mathrm{~Hz}\right), 2.93(\mathrm{~d}, 1 \mathrm{H}, 4-\mathrm{H}, J=4.6 \mathrm{~Hz}), 2.87\left(\mathrm{~s}, 6 \mathrm{H}, \mathrm{SO}_{2} \mathrm{NMe}_{2}\right), 2.39-2.33\left(\mathrm{~m}, 1 \mathrm{H}, 6-\mathrm{H}_{\text {exo }}\right.$ ), 2.16-2.11 (m, 1H, 5- $\left.\mathrm{H}_{\text {exo }}\right), 1.74-1.69\left(\mathrm{~m}, 1 \mathrm{H}, 5-\mathrm{H}_{\text {endo }}\right), 1.65-1.60\left(\mathrm{~m}, 1 \mathrm{H}, 6-\mathrm{H}_{\text {endo }}\right), 1.22(\mathrm{~s}, 3 \mathrm{H}, 8-\mathrm{H}), 1.21(\mathrm{~s}, 3 \mathrm{H}, 9-\mathrm{H})$. ${ }^{13} \mathrm{C} \mathrm{NMR}\left(150.92 \mathrm{MHz}, \mathrm{CDCl}_{3}, 293 \mathrm{~K}\right): \delta=176.57$ (1C, $\left.1^{`}-\mathrm{C}\right), 152.43$ (1C, 2`-C), 148.47 (1C, 3-C), 135.65 (1C, $\left.7{ }^{`}-\mathrm{C}\right)$, 126.23 (1C, $\left.4^{`}-\mathrm{C}\right), 125.32$ (1C, $\left.5^{`}-\mathrm{C}\right), 124.72$ (1C, 11-C), 122.92 (1C, 6`C), 121.57 (1C, $\left.3^{`}-\mathrm{C}\right), 84.54$ (1C, 2-C), 81.72 (1C, 12-C), 69.80 (1C, 13-C), 68.95 (2C, 14-C, 15-C ), 68.87 (5C, Cp), 67.86 (1C, 16-C), 55.53 (1C, 1-C), 51.13 (1C, 4C), 50.41 (1C, 7-C), 43.65 (1C, 10-C), 37.61 (2C, $\mathrm{SO}_{2} \mathrm{NMe}_{2}$ ), 27.04 (1C, 6-C), 25.03 (1C, 5-C), 22.06 (1C, 8-C), 21.15 (1C, 9-C). MS (CI) m/z (rel. int.): 591 (M+1, 51), 590 (M, 61), $573\left(\mathrm{M}-\mathrm{H}_{2} \mathrm{O}, 100\right), 136$ (benzothiazole+1, 28). Anal. calcd. for $\mathrm{C}_{30} \mathrm{H}_{34} \mathrm{FeN}_{2} \mathrm{O}_{3} \mathrm{~S}_{2}$ (590.58): C, 61.01; H, 5.80; Fe, 9.46; N, 4.74; S, 10.86. Found: C, 60.96; H 5.70; Fe, 9.62; $\mathrm{N}, 4.63 ; \mathrm{S}, 10.97 \%$.

\subsection{2 -((1R,2R,4S,E)-3-Ferrocenylmethylidene-2-hydroxy-1,7,7-trimethylbicyclo[2.2.1]heptan-2-yl)acetonitrile 40}

To a solution of dry acetonitrile $(0.177 \mathrm{~g}, 4.31 \mathrm{mmol})$ in $20 \mathrm{ml}$ of THF was added $n$-BuLi $(1.72 \mathrm{ml}, 4.31 \mathrm{mmol}$ of $2.5 \mathrm{M}$ solution in hexane) dropwise at $-78^{\circ} \mathrm{C}$. After stirring for $45 \mathrm{~min}$ at $-78^{\circ} \mathrm{C}$, compound $7(1.00 \mathrm{~g}, 2.87 \mathrm{mmol}) \mathrm{was}$ added and the reaction mixture was stirred for $3 \mathrm{~h}$ while keeping the temperature between $-78^{\circ} \mathrm{C}$ and $-65^{\circ} \mathrm{C}$. The reaction progress was monitored by TLC (petroleum ether/MTBE $=7: 1$ ). The mixture was quenched at this temperature with saturated aqueous $\mathrm{NH}_{4} \mathrm{Cl}$, after which the mixture warmed to room temperature, extracted with $\mathrm{Et}_{2} \mathrm{O}$ and the organic phase washed with water, and dried over anhydrous $\mathrm{Na}_{2} \mathrm{SO}_{4}$. The solvent was evaporated in vacuo and the crude product was chromatographed (190 g silica gel, petroleum ether/MTBE $=7: 1)$, to give $\mathbf{4 0}(0.711 \mathrm{~g}, 64 \%)$ as an orange solid. Mp 115-117 ${ }^{\circ} \mathrm{C} .[\alpha]_{\mathrm{D}}^{20}=-49.0\left(c 0.74, \mathrm{CHCl}_{3}\right) .{ }^{1} \mathrm{H} \mathrm{NMR}\left(600 \mathrm{MHz}, \mathrm{CDCl}_{3}, 293 \mathrm{~K}\right): \delta=6.46(\mathrm{~s}, 1 \mathrm{H}, 11-\mathrm{H}), 4.33-$ 4.32 (m, 2H, 16-H, 13-H), 4.25-4.24* (m, 1H, 15-H), 4.23-4.22* (m, 1H, 14-H), 4.13 (s, 5H, Cp), 2.82 (d, 1H, 4-H, J= $4.6 \mathrm{~Hz}), 2.69\left(\mathrm{~d}, 1 \mathrm{H}, 1^{`}-\mathrm{H}_{\mathrm{a}}, J=16.6 \mathrm{~Hz}\right), 2.65\left(\mathrm{~d}, 1 \mathrm{H}, 1 \mathrm{C}^{`} \mathrm{H}_{\mathrm{b}}, J=16.6 \mathrm{~Hz}\right), 1.99-1.94\left(\mathrm{~m}, 2 \mathrm{H}, 5-\mathrm{H}_{\text {exo }}, \mathrm{OH}\right), 1.70(\mathrm{ddd}$, $\left.1 \mathrm{H}, 6-\mathrm{H}_{\text {exo }}, J=14.1,11.8,5.4 \mathrm{~Hz}\right), 1.44-1.40\left(\mathrm{~m}, 1 \mathrm{H}, 6-\mathrm{H}_{\text {endo }}\right), 1.31-1.26\left(\mathrm{~m}, 1 \mathrm{H}, 5-\mathrm{H}_{\text {endo }}\right), 1.07(\mathrm{~s}, 3 \mathrm{H}, 10-\mathrm{H}), 1.02(\mathrm{~s}$, $3 \mathrm{H}, 8-\mathrm{H}), 0.94$ (s, 3H, 9-H). ${ }^{13} \mathrm{C}$ NMR (150.92 MHz, $\left.\mathrm{CDCl}_{3}, 293 \mathrm{~K}\right): \delta=148.94$ (1C, 3-C), 119.81 (1C, 11-C), 118.41 $(1 \mathrm{C}, \mathrm{CN}), 78.56(2 \mathrm{C}, 2-\mathrm{C}, 12-\mathrm{C}), 69.43^{*}(1 \mathrm{C}, 16-\mathrm{C}), 69.01^{*}(1 \mathrm{C}, 15-\mathrm{C}), 68.82(5 \mathrm{C}, \mathrm{Cp}), 68.74^{*}(1 \mathrm{C}, 14-\mathrm{C}), 68.09^{*}(1 \mathrm{C}$, 13-C), 53.03 (1C, 7-C), 50.85 (1C, 4-C), 48.53 (1C, 1-C), 31.00 (1C, 6-C), 28.72 (1C, 1`-C), 24.75 (1C, 5-C), 22.23 
(1C, 8-C), 19.95 (1C, 9-C), 9.90 (1C, 10-C). MS (ESI) m/z (rel. int.): 390 (M+1, 6), 372 (M-H 2 O, 100). Anal. calcd. for $\mathrm{C}_{23} \mathrm{H}_{27} \mathrm{FeNO}$ (389.31): C, 70.96; H, 6.99; Fe, 14.34; N, 3.60. Found: C, 70.89; H 7.07; Fe, 14.42; N, $3.69 \%$.

4.17. 1-((1S,2R,4S,E)-2-(Cyanomethyl)-3-ferrocenylmethylidene-2-hydroxy-7,7-dimethylbicyclo[2.2.1]heptan-1yl)- $N, N$-dimethylmethanesulfonamide 41

To a solution of dry acetonitrile $(0.108 \mathrm{~g}, 2.64 \mathrm{mmol})$ in $10 \mathrm{ml}$ of THF was added $n$-BuLi $(0.88 \mathrm{ml}, 2.20 \mathrm{mmol}$ of $2.5 \mathrm{M}$ solution in hexane) dropwise at $-78^{\circ} \mathrm{C}$. After stirring for $45 \mathrm{~min}$ at $-78^{\circ} \mathrm{C}$, compound $9(0.500 \mathrm{~g}, 1.10 \mathrm{mmol})$ was added and the reaction mixture was stirred for $3 \mathrm{~h}$ while keeping the temperature between $-78^{\circ} \mathrm{C}$ and $-65^{\circ} \mathrm{C}$. The reaction progress was monitored by $\mathrm{TLC}$ (hexane/ $\mathrm{Et}_{2} \mathrm{O}=5: 1$ ). The reaction was quenched at this temperature with saturated aq. $\mathrm{NH}_{4} \mathrm{Cl}$, the mixture warmed to room temperature, extracted with $\mathrm{Et}_{2} \mathrm{O}$ and the organic phase washed with water, and dried over anhydrous $\mathrm{Na}_{2} \mathrm{SO}_{4}$. The solvent was evaporated in vacuo and the crude product was chromatographed ( $8 \mathrm{~g}$ silica gel, hexane/ $\left.\mathrm{Et}_{2} \mathrm{O}=5: 1\right)$, to give $41(0.502 \mathrm{~g}, 92 \%)$ as an orange solid. $\mathrm{Mp} 125-128^{\circ} \mathrm{C}$. $[\alpha]_{\mathrm{D}}^{20}=-98.7\left(c 0.54, \mathrm{CHCl}_{3}\right) .{ }^{1} \mathrm{H} \mathrm{NMR}\left(600 \mathrm{MHz}, \mathrm{CDCl}_{3}, 293 \mathrm{~K}\right): \delta=6.73(\mathrm{~s}, 1 \mathrm{H}, 11-\mathrm{H}), 4.34^{*}$ (br s, 1H, 13-H), 4.32* (br s, 1H, 16-H), 4.24* (br s, 1H, 14-H), 4.22* (br s, 1H, 15-H), 4.17 (s, 5H, Cp), 3.61 (d, 1H, 1 - $\left.-\mathrm{H}_{\mathrm{a}}, J=16.7 \mathrm{~Hz}\right), 3.38$ $\left(\mathrm{d}, 1 \mathrm{H}, 10-\mathrm{H}_{\mathrm{a}}, J=14.1 \mathrm{~Hz}\right), 2.93\left(\mathrm{~s}, 6 \mathrm{H}, \mathrm{SO}_{2} \mathrm{NMe}_{2}\right), 2.80(\mathrm{~d}, 1 \mathrm{H}, 4-\mathrm{H}, J=4.8 \mathrm{~Hz}), 2.76\left(\mathrm{~d}, 1 \mathrm{H}, 1-\mathrm{H}_{\mathrm{b}}, J=16.7 \mathrm{~Hz}\right), 2.68$ $\left(\mathrm{d}, 1 \mathrm{H}, 10-\mathrm{H}_{\mathrm{a}}, J=14.1 \mathrm{~Hz}\right), 2.07-2.02\left(\mathrm{~m}, 1 \mathrm{H}, 5-\mathrm{H}_{\text {exo }}\right), 1.97-1.88\left(\mathrm{~m}, 2 \mathrm{H}, 6-\mathrm{H}_{\text {exo }}, 6-\mathrm{H}_{\text {endo }}\right), 1.49-1.45\left(\mathrm{~m}, 1 \mathrm{H}, 5-\mathrm{H}_{\text {endo }}\right)$, 1.02 (s, 3H, 8-H), 0.93 (s, 3H, 9-H). $\left.{ }^{13} \mathrm{C} \mathrm{NMR} \mathrm{(150.92} \mathrm{MHz,} \mathrm{CDCl}_{3}, 293 \mathrm{~K}\right): \delta=146.34$ (1C, 3-C), 120.18 (1C, 11-C), $118.38(1 \mathrm{C}, \mathrm{CN}), 81.73(1 \mathrm{C}, 2-\mathrm{C}), 77.12(1 \mathrm{C}, 12-\mathrm{C}) 69.63^{*}(1 \mathrm{C}, 13-\mathrm{C}), 69.09$ (5C, Cp), 68.76 ${ }^{*}(1 \mathrm{C}, 14-\mathrm{C}), 68.65^{*}(1 \mathrm{C}$, 15-C), 67.87* (1C, 16-C), 54.20 (1C, 7-C), 51.20 (1C, 1-C), 50.02 (1C, 4-C), 45.17 (1C, 10-C), 37.60 $\left(2 \mathrm{C}, \mathrm{SO}_{2} \mathrm{NMe}_{2}\right)$, 30.13 (1C, 1-C), 27.75 (1C, 6-H), 24.95 (1C, 5-H), 21.74 (1C, 8-C), 20.09 (1C, 9-C). MS (ESI) m/z (rel. int.): 497 $(\mathrm{M}+1,90), 480\left(\mathrm{M}-\mathrm{H}_{2} \mathrm{O}, 100\right)$. Anal. calcd. for $\mathrm{C}_{25} \mathrm{H}_{32} \mathrm{FeN}_{2} \mathrm{O}_{3} \mathrm{~S}$ (496.44): C, 60.48; H, 6.50; Fe, 11.25; N, 5.64; S, 6.46. Found: C, 60.36; H 6.58; Fe, 11.39; N, 5.56; S, $6.52 \%$.

\subsection{8. (1R,2R,4S,E)-2-(2-Aminoethyl)-3-ferrocenylmethylidene-1,7,7-trimethylbicyclo[2.2.1]heptan-2-ol 42}

To a suspension of $\mathrm{LiAlH}_{4}(0.486 \mathrm{~g}, 12.80 \mathrm{mmol})$ in $25 \mathrm{ml}$ of $\mathrm{Et}_{2} \mathrm{O}$ was added dropwise a solution of 40 (0.500 $\mathrm{g}, 1.28 \mathrm{mmol}$ ) in $10 \mathrm{ml}$ of $\mathrm{THF}$ at $0^{\circ} \mathrm{C}$ within $30 \mathrm{~min}$ and the resulting mixture was stirred for $1 \mathrm{~h}$. The reaction progress was monitored by TLC (petroleum ether/MTBE $=50: 1$ ). The reaction mixture was carefully quenched with $\mathrm{H}_{2} \mathrm{O}$ at $0^{\circ} \mathrm{C}$ and the mixture was stirred for $30 \mathrm{~min}$. The inorganic salts were filtered and washed with $\mathrm{Et}_{2} \mathrm{O}$ and $\mathrm{CH}_{2} \mathrm{Cl}_{2}$. The combined organic phase was washed with water and dried over anhydrous $\mathrm{Na}_{2} \mathrm{SO}_{4}$. The solvent was evaporated in vacuo and the crude product was purified by chromatographic filtration $\left(3 \mathrm{~g}\right.$ basic $\mathrm{Al}_{2} \mathrm{O}_{3}$, petroleum ether/MTBE $=50: 1$ to MTBE), to give $42(0.500 \mathrm{~g}, 99 \%)$ as an orange solid. $\mathrm{Mp} 83-85^{\circ} \mathrm{C}$. $[\alpha]_{\mathrm{D}}^{20}=-20.5\left(c 0.44, \mathrm{CHCl}_{3}\right)$. ${ }^{1} \mathrm{H} \mathrm{NMR}(600$ $\left.\mathrm{MHz} \mathrm{CDCl}_{3}, 293 \mathrm{~K}\right): \delta=6.15(\mathrm{~s}, 1 \mathrm{H}, 11-\mathrm{H}), 4.32-4.31(\mathrm{~m}, 2 \mathrm{H}, 13-\mathrm{H}, 16-\mathrm{H}), 4.18-4.17^{*}$ (m, 1H, 14-H), 4.16-4.15* (m, $1 \mathrm{H}, 15-\mathrm{H}), 4.09$ (s, 5H, Cp), 3.18-3.14 (m, 1H, 2- $\left.\mathrm{H}_{\mathrm{b}}\right), 3.11-3.06\left(\mathrm{~m}, 1 \mathrm{H}, 2-\mathrm{H}_{\mathrm{a}}\right), 2.72(\mathrm{~d}, 1 \mathrm{H}, 4-\mathrm{H}, J=4.8 \mathrm{~Hz}), 1.94-$ $1.88\left(\mathrm{~m}, 1 \mathrm{H}, 5-\mathrm{H}_{\text {exo }}\right), 1.79-1.77(\mathrm{~m}, 2 \mathrm{H}, 1 `-\mathrm{H}), 1.62-1.57\left(\mathrm{~m}, 1 \mathrm{H}, 6-\mathrm{H}_{\text {exo }}\right), 1.49-1.45\left(\mathrm{~m}, 1 \mathrm{H}, 6-\mathrm{H}_{\text {endo }}\right), 1.26-1.21(\mathrm{~m}, 2 \mathrm{H}$, $\left.5-\mathrm{H}_{\text {endo }}, \mathrm{OH}\right), 1.03(\mathrm{~s}, 3 \mathrm{H}, 10-\mathrm{H}), 1.01(\mathrm{~s}, 3 \mathrm{H}, 8-\mathrm{H}), 0.89(\mathrm{~s}, 3 \mathrm{H}, 9-\mathrm{H}) .{ }^{13} \mathrm{C} \mathrm{NMR}\left(150.92 \mathrm{MHz}, \mathrm{CDCl}_{3}, 293 \mathrm{~K}\right): \delta=$ 153.63 (1C, 3-C), 116.90 (1C, 11-C), 83.57 (1C, 12-C), 82.41 (1C, 2-C), 68.97* (1C, 16-C), 68.84 (5C, Cp), $68.16(2 \mathrm{C}$, 14-C, 15-C), 68.02* (1C, 13-C), 52.52 (1C, 7-C), 50.88 (1C, 4-C), 49.18 (1C, 1-C), 41.16 (1C, $\left.1^{`}-\mathrm{C}\right), 39.18$ (1C, $\left.22^{`}-\mathrm{C}\right)$, 30.61 (1C, 6-C), 25.29 (1C, 5-C), 22.20 (1C, 8-C), 20.02 (1C, 9-C), 11.96 (1C, 10-C). MS (ESI) m/z (rel. int.): 394 $(\mathrm{M}+1,5), 377\left(\mathrm{M}-\mathrm{H}_{2} \mathrm{O}, 100\right)$. Anal. calcd. for $\mathrm{C}_{23} \mathrm{H}_{31} \mathrm{FeNO}$ (393.34): C, 70.23; H, 7.94; Fe, 14.20; N, 3.56. Found: C, 70.34; H 7.99; Fe, 14.34; N, $3.44 \%$.

\subsection{1-((1S,2R,4S,E)-2-(2-Aminoethyl)-3-ferrocenylmethylidene-2-hydroxy-7,7-dimethylbicyclo[2.2.1]heptan-1- yl)- $N, N$-dimethylmethanesulfonamide 43}

To a suspension of $\mathrm{LiAlH}_{4}(0.920 \mathrm{~g}, 24.20 \mathrm{mmol})$ in $100 \mathrm{ml}$ of $\mathrm{Et}_{2} \mathrm{O}$ was added dropwise a solution of 41 $(1.200 \mathrm{~g}, 2.42 \mathrm{mmol})$ in $30 \mathrm{ml}$ of THF at $0^{\circ} \mathrm{C}$ within $30 \mathrm{~min}$, and the resulting mixture was stirred for $1 \mathrm{~h}$. The reaction progress was monitored by TLC $\left(\mathrm{Et}_{2} \mathrm{O} / \mathrm{MeOH} / \mathrm{Et}_{3} \mathrm{~N}=100: 2: 0.1\right)$. The reaction mixture was carefully quenched with $\mathrm{H}_{2} \mathrm{O}$ at $0^{\circ} \mathrm{C}$ and the mixture was stirred for 30 min. The inorganic salts were filtered and washed with $\mathrm{Et}_{2} \mathrm{O}$, and $\mathrm{CH}_{2} \mathrm{Cl}_{2}$. The combined organic phase was washed with water and dried over anhydrous $\mathrm{Na}_{2} \mathrm{SO}_{4}$. The solvent was evaporated in vacuo and the crude product was chromatographed (100 g silica gel, $\mathrm{Et}_{2} \mathrm{O} / \mathrm{MeOH} / \mathrm{Et}_{3} \mathrm{~N}=100: 2: 0.1$ to 100:10:0.1), to give $43(0.736 \mathrm{~g}, 61 \%)$ as orange solid. $\mathrm{Mp} 88-89^{\circ} \mathrm{C}$. $[\alpha]_{\mathrm{D}}^{20}=-28.2\left(c 0.54, \mathrm{CHCl}_{3}\right) .{ }^{1} \mathrm{H} \mathrm{NMR}\left(600 \mathrm{MHz}, \mathrm{CDCl}_{3}, 293 \mathrm{~K}\right)$ : $\delta=6.26(\mathrm{~s}, 1 \mathrm{H}, 11-\mathrm{H}), 4.30-4.29(\mathrm{~m}, 2 \mathrm{H}, 13-\mathrm{H}, 16-\mathrm{H}), 4.19-4.18^{*}(\mathrm{~m}, 1 \mathrm{H}, 14-\mathrm{H}), 4.17-4.16^{*}(\mathrm{~m}, 1 \mathrm{H}, 15-\mathrm{H}), 4.09(\mathrm{~s}, 5 \mathrm{H}$, $\mathrm{Cp}), 3.59$ (d, $\left.1 \mathrm{H}, 10-\mathrm{H}_{\mathrm{b}}, J=14.4 \mathrm{~Hz}\right), 3.33-3.24(\mathrm{~m}, 2 \mathrm{H}, 2-\mathrm{H}), 2.90$ (s, 6H, $\left.\mathrm{SO}_{2} \mathrm{NMe}_{2}\right), 2.74$ (d, 1H, 4-H, $\left.J=4.8 \mathrm{~Hz}\right)$, $2.68\left(\mathrm{~d}, 1 \mathrm{H}, 10-\mathrm{H}_{\mathrm{a}}, J=14.4 \mathrm{~Hz}\right), 2.39-2.34^{*}\left(\mathrm{~m}, 1 \mathrm{H}, 1-\mathrm{H}_{\mathrm{a}}\right), 2.16-2.11\left(\mathrm{~m}, 1 \mathrm{H}, 6-\mathrm{H}_{\text {exo }}\right), 1.99-1.92\left(\mathrm{~m}, 2 \mathrm{H}, 5-\mathrm{H}_{\text {exo }}, 6-\right.$ $\mathrm{H}_{\text {endo }}$ ), 1.64-1.60* (m, 1H, 1 $\left.-\mathrm{H}_{\mathrm{b}}\right), 1.35-1.30\left(\mathrm{~m}, 1 \mathrm{H}, 5-\mathrm{H}_{\text {endo }}\right), 0.99$ (s, 3H, 8-H), $0.96(\mathrm{~s}, 3 \mathrm{H}, 9-\mathrm{H}) .{ }^{13} \mathrm{C} \mathrm{NMR}(150.92$ $\left.\mathrm{MHz}, \mathrm{CDCl}_{3}, 293 \mathrm{~K}\right): \delta=152.74$ (1C, 3-C), 116.88 (1C, 11-C), 83.32 (1C, 12-C), 81.81 (1C, 2-C), 68.94* (1C, 16-C), 68.80 (5C, Cp), 68.26* (1C, 13-C), 68.20* (1C, 15-C), 68.09* (1C, 14-C), 53.68 (1C, 7-C), 50.72 (1C, 1-C), 50.31 (1C, 4-C), 43.80 (1C, 10-C), 38.32 (1C, 2`C), 37.74 (1C, 1`-C), 37.72 (2C, $\mathrm{SO}_{2} \mathrm{NMe}_{2}$ ), 26.13 (1C, 6-C), 24.99 (1C, 5-C), 
21.81 (1C, 8-C), 20.33 (1C, 9-C). MS (ESI) m/z (rel. int.): 501 (M+1, 30), 484 (M- $\left.\mathrm{H}_{2} \mathrm{O}, 48\right), 455$ (M-EtNH $\left.{ }_{2}, 100\right)$. Anal. calcd. for $\mathrm{C}_{25} \mathrm{H}_{36} \mathrm{FeN}_{2} \mathrm{O}_{3} \mathrm{~S}$ (500.47): C, 60.00; H, 7.25; Fe, 11.16; N, 5.60; S, 6.41. Found: C, 60.09; H 7.35; Fe, 11.08; N, 5.69; S, $6.35 \%$.

\subsection{0. (1R,2R,4S,E)-3-Ferrocenylmethylidene-2-(2-(isoindolin-2-yl)ethyl)-1,7,7-trimethylbicyclo[2.2.1]heptan-2-ol 45}

To a stirred solution of $42(0.350 \mathrm{~g}, 0.89 \mathrm{mmol})$ in $\mathrm{CH}_{3} \mathrm{CN}(25 \mathrm{ml})$ were added $\mathrm{K}_{2} \mathrm{CO}_{3}(0.160 \mathrm{~g}, 1.16 \mathrm{mmol})$ and $\alpha, \alpha^{\prime}$-dichloro-o-xylene $(0.187 \mathrm{~g}, 1.07 \mathrm{mmol})$ at room temperature. The reaction mixture was refluxed for $17 \mathrm{~h}$. The reaction progress was monitored by TLC (petroleum ether/MTBE $=2: 1$ ). The mixture was quenched with $\mathrm{H}_{2} \mathrm{O}$, extracted with $\mathrm{CH}_{2} \mathrm{Cl}_{2}$ and the organic phase was dried over anhydrous $\mathrm{Na}_{2} \mathrm{SO}_{4}$. The solvent was evaporated in vacuo and the crude product was chromatographed (40 g silica gel, petroleum ether/MTBE $=2: 1$ to $1: 1)$, to give $45(0.110 \mathrm{~g}$, $25 \%)$ as orange solid. Mp 126-128 ${ }^{\circ} \mathrm{C} .[\alpha]_{\mathrm{D}}^{20}=+51.3\left(c 0.39, \mathrm{CHCl}_{3}\right) .{ }^{1} \mathrm{H} \mathrm{NMR}\left(600 \mathrm{MHz}, \mathrm{CDCl}_{3}, 293 \mathrm{~K}\right): \delta=7.20(\mathrm{~s}$, $\left.4 \mathrm{H}, 5^{`}-\mathrm{H}, 6^{\prime}-\mathrm{H}, 7^{`}-\mathrm{H}, 8^{`}-\mathrm{H}\right), 6.19$ (s, 1H, 11-H), 4.34-4.33 (m, 2H, 13-H, 16-H), 4.19-4.16* (m, 4H, 14-H, 15-H, 10`-H), 4.12 (s, 5H, Cp), 3.99* (d, 2H, 3`- H, J = 11.6 Hz), 3.28-3.23 (m, 1H, 2`- $\mathrm{H}_{\mathrm{a}}$ ), 3.02-2.99 (m, 1H, 2`- $\left.\mathrm{H}_{\mathrm{b}}\right), 2.74(\mathrm{~d}, 1 \mathrm{H}, 4-\mathrm{H}$, $J=4.7 \mathrm{~Hz}), 1.99-1.92\left(\mathrm{~m}, 2 \mathrm{H}, 5-\mathrm{H}_{\text {exo }}, 1^{\prime}-\mathrm{H}_{\mathrm{a}}\right), 1.87-1.83\left(\mathrm{~m}, 1 \mathrm{H}, 1-\mathrm{H}_{\mathrm{b}}\right), 1.66-1.61\left(\mathrm{~m}, 1 \mathrm{H}, 6-\mathrm{H}_{\text {exo }}\right), 1.53-1.49(\mathrm{~m}, 1 \mathrm{H}, 6-$ $\mathrm{H}_{\text {endo }}$ ), 1.29-1.25 (m, 1H, 5- $\left.\mathrm{H}_{\text {endo }}\right), 1.07$ (s, 3H, 10-H), 0.99 (s, 3H, 8-H), $0.90(\mathrm{~s}, 3 \mathrm{H}, 9-\mathrm{H}) .{ }^{13} \mathrm{C} \mathrm{NMR}(150.92 \mathrm{MHz}$, $\left.\mathrm{CDCl}_{3}, 293 \mathrm{~K}\right): \delta=153.39$ (1C, 3-C), 139.42 (2C, $\left.4^{`}-\mathrm{C}, 9^{`}-\mathrm{C}\right), 126.85^{*}$ (2C, $\left.5^{`}-\mathrm{C}, 8^{`}-\mathrm{C}\right), 122.22^{*}$ (2C, $\left.6^{`}-\mathrm{C}, 7^{`}-\mathrm{C}\right)$, 116.97 (1C, 11-C), 83.58 (1C, 12-C), 82.58 (1C, 2-C), 69.04* (1C, 15-C), 68.86 (5C, Cp), 68.18 (2C, 13-C, 16-C), $68.01^{*}$ (1C, 14-C), 59.20 (2C, $\left.3^{`}-\mathrm{C}, 10^{`}-\mathrm{C}\right), 53.89$ (1C, $\left.2^{`}-\mathrm{C}\right), 52.46$ (1C, 7-C), 50.93 (1C, 4-C), 49.26 (1C, 1-C), 37.02 (1C, 1`C), 30.68 (1C, 6-C), 25.35(1C, 5-C), 22.11 (1C, 8-C), 20.03 (1C, 9-C), 12.06 (1C, 3-C). MS (ESI) m/z (rel. int.): $496(\mathrm{M}+1,16), 479\left(\mathrm{M}-\mathrm{H}_{2} \mathrm{O}, 13\right), 132$ (N-methylisoindoline, 100). Anal. calcd. for $\mathrm{C}_{31} \mathrm{H}_{37} \mathrm{FeNO}$ (495.48): C, 75.15; H, 7.53; Fe, 11.27; N, 2.83. Found: C, 75.08; H 7.48; Fe, 11.36; N, $2.91 \%$.

\subsection{1. \\ 1-((1S,2R,4S,E)-3-Ferrocenylmethylidene-2-hydroxy-2-(2-(isoindolin-2-yl)ethyl)-7,7- dimethylbicyclo[2.2.1] heptan-1-yl)- $N, N$-dimethylmethanesulfonamide 46}

To a stirred solution of $43(0.300 \mathrm{~g}, 0.60 \mathrm{mmol})$ in $\mathrm{CH}_{3} \mathrm{CN}(15 \mathrm{ml})$ were added $\mathrm{K}_{2} \mathrm{CO}_{3}(0.133 \mathrm{~g}, 0.96 \mathrm{mmol})$ and $\alpha, \alpha^{\prime}$-dichloro-o-xylene $(0.158 \mathrm{~g}, 0.90 \mathrm{mmol})$ at room temperature. The reaction mixture was refluxed for $7 \mathrm{~h}$. The reaction progress was monitored by TLC (cyclohexane/MTBE $=2: 1$ ). The reaction was quenched with water, extracted with $\mathrm{CH}_{2} \mathrm{Cl}_{2}$ and the organic phase was dried over anhydrous $\mathrm{Na}_{2} \mathrm{SO}_{4}$. The solvent was evaporated in vacuo and the crude product was chromatographed (30 g silica gel, cyclohexane/MTBE $=2: 1)$, to give $46(0.097 \mathrm{~g}, 27 \%)$ as an orange

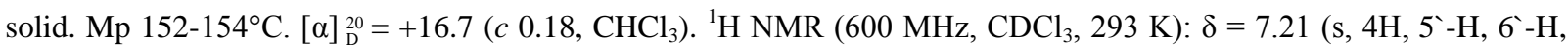
$\left.7-\mathrm{H}, 8^{`}-\mathrm{H}\right), 6.26(\mathrm{~s}, 1 \mathrm{H}, 11-\mathrm{H}), 4.33-4.32(\mathrm{~m}, 2 \mathrm{H}, 13-\mathrm{H}, 16-\mathrm{H}), 4.22-4.18^{*}(\mathrm{~m}, 4 \mathrm{H}, 14-\mathrm{H}, 15-\mathrm{H}, 10 `-\mathrm{H}), 4.13(\mathrm{~s}, 5 \mathrm{H}$, $\mathrm{Cp}), 4.06^{*}(\mathrm{br} \mathrm{d}, 2 \mathrm{H}, 3-\mathrm{H}, J=11.8 \mathrm{~Hz}), 3.63\left(\mathrm{~d}, 1 \mathrm{H}, 10-\mathrm{H}_{\mathrm{b}}, J=14.9 \mathrm{~Hz}\right), 3.55\left(\mathrm{dt}, 1 \mathrm{H}, 2^{-}-\mathrm{H}_{\mathrm{a}}, J=12.2,2.7 \mathrm{~Hz}\right), 3.07-$ $3.04\left(\mathrm{~m}, 1 \mathrm{H}, 2-\mathrm{H}_{\mathrm{b}}\right), 2.94\left(\mathrm{~s}, 6 \mathrm{H}, \mathrm{SO}_{2} \mathrm{NMe}_{2}\right), 2.76(\mathrm{~d}, 1 \mathrm{H}, 4-\mathrm{H}, J=4.7 \mathrm{~Hz}), 2.70-2.65\left(\mathrm{~m}, 2 \mathrm{H}, 10-\mathrm{H}_{\mathrm{a}}, 1-\mathrm{H}_{\mathrm{a}}\right), 2.26-2.21$ $\left(\mathrm{m}, 1 \mathrm{H}, 6-\mathrm{H}_{\text {exo }}\right), 2.02-1.96\left(\mathrm{~m}, 2 \mathrm{H}, 5-\mathrm{H}_{\text {exo }}, 6-\mathrm{H}_{\text {endo }}\right), 1.62-1.58\left(\mathrm{~m}, 1 \mathrm{H}, 1-\mathrm{H}_{\mathrm{b}}\right), 1.38-1.34\left(\mathrm{~m}, 1 \mathrm{H}, 5-\mathrm{H}_{\text {endo }}\right), 0.97(\mathrm{~s}, 3 \mathrm{H}, 9-$ $\mathrm{H}), 0.95$ (s, 3H, 8-H). ${ }^{13} \mathrm{C}$ NMR (150.92 MHz, $\left.\mathrm{CDCl}_{3}, 293 \mathrm{~K}\right): \delta=152.55$ (1C, 3-C), 139.37 (2C, $\left.4^{-}-\mathrm{C}, 9^{`}-\mathrm{C}\right), 126.91$ (2C, $\left.5^{`}-\mathrm{C}, 8^{`}-\mathrm{C}\right), 122.19$ (2C, $\left.6^{`}-\mathrm{C}, 7^{-}-\mathrm{C}\right), 117.33$ (1C, 11-C), 83.31 (1C, 12-C), 81.83 (1C, 2-C), 68.98* (1C, 13-C), 68.85 (5C, Cp), 68.35* (1C, 16-C), 68.33* (1C, 14-C), 68.14* (1C, 15-C), 59.00 (2C, 3`-C, 10`C), 53.58 (1C, 7-C), 52.55 (1C, $\left.2{ }^{-}-\mathrm{C}\right), 50.70$ (1C, 1-C), 50.44 (1C, 4-C), 43.63 (1C, 10-C), 37.76 (2C, $\left.\mathrm{SO}_{2} \mathrm{NMe}_{2}\right), 33.60$ (1C, $\left.1^{`}-\mathrm{C}\right), 25.80$ (1C, 6-C), 24.90 (1C, 5-C), 21.67 (1C, 8-C), 20.34 (1C, 9-C). MS (ESI) m/z (rel. int.): 603 (M+1, 38), 585 (M- $\left.\mathrm{H}_{2} \mathrm{O}, 7\right)$, 132 (N-methylisoindoline, 100). Anal. calcd. for $\mathrm{C}_{33} \mathrm{H}_{42} \mathrm{FeN}_{2} \mathrm{O}_{3} \mathrm{~S}$ (602.61): C, 65.77; H, 7.03; Fe, 9.27; N, 4.65; S, 5.32. Found: C, 65.85; H 6.98; Fe, 9.21; N, 4.62; S, $5.23 \%$.

\subsection{Enantioselective addition of diethylzinc to benzaldehyde in presence of ligands $15-19,29-35,45$ and 46} (General procedure)

To a solution of the corresponding ligand in $6 \mathrm{ml}$ of hexane or toluene, $\mathrm{Et}_{2} \mathrm{Zn}(5.6 \mathrm{ml}, 5.60 \mathrm{mmol}$ of $1 \mathrm{M}$ solution in hexane) was added dropwise at $0^{\circ} \mathrm{C}$. The mixture was stirred for $30 \mathrm{~min}$ at $0^{\circ} \mathrm{C}$ and then benzaldehyde $(0.288 \mathrm{ml}, 2.83 \mathrm{mmol})$ was added at $0^{\circ} \mathrm{C}$. The reaction was stirred at room temperature and monitored by TLC (petroleum ether $/ \mathrm{Et}_{2} \mathrm{O}=5: 1$ ) until the full consumption of benzaldehyde. The mixture was quenched at $0^{\circ} \mathrm{C}$ (aq. $\left.\mathrm{NH}_{4} \mathrm{Cl}\right)$, extracted with $\mathrm{Et}_{2} \mathrm{O}(3 \times 20 \mathrm{ml})$ and organic phase was dried over anhydrous $\mathrm{Na}_{2} \mathrm{SO}_{4}$. The solvent was evaporated in vacuo and the crude product was chromatographed $\left(15 \mathrm{~g}\right.$ silica gel, petroleum ether/Et $\left.{ }_{2} \mathrm{O}=6: 1\right)$ to obtain pure 1-phenyl-1-propanol as a mixture of both enantiomers. For the ee determination of 1-phenyl-1-propanol, see section 4.1. General.

\section{Acknowledgements}

Support of this work by the Bulgarian Scientific Fund (project MU0135/2008), by SCOPES program of Swiss National Science Foundation (project IB7320-111072/1) and by the Alexander von Humboldt Foundation is gratefully 
acknowledged. Support by the National Science Fund of Bulgaria (projects DID02/33/2009, DRNF 02/1/2009, UNA 17/2005 and DRNF 02/13/2009) is acknowledged.

Keywords: Asymmetric synthesis, (+)-camphor, (+)-camphor-10-sulfonamide, organolithium, ferrocene, enantioselectivity, diethylzinc

\section{References and notes:}

1. $\quad$ Oguni, N.; Omi, T. Tetrahedron Letters 1984, 25, 2823-2824.

Nugent, W. A. Advanced Synthesis and Catalysis 2003, 345, 415-424.

Chavez, D. E.; Jacobsen, E. N. Angewandte Chemie - International Edition 2001, 40, 3667-3670.

Feringa, B. L. Accounts of Chemical Research 2000, 33, 346-353.

Fukuda, A.; Kobayashi, Y.; Kimachi, T.; Takemoto, Y. Tetrahedron 2003, 59, 9305-9313.

Knochel, P.; Jones, P. Organozinc Reagents: A Practical Approach; Oxford University Press, 1999.

Knochel, P. Handbook of Functionalized Organometallics: Applications in Synthesis; Wiley, 2005.

Rappoport, Z.; Marek, I. The Chemistry of Organozinc Compounds: R-Zn; Wiley, 2007.

$\mathrm{Pu}, \mathrm{L}$; $\mathrm{Yu}, \mathrm{H}$. B. Chemical Reviews 2001, 101, 757-824.

Dimitrov, V.; Kostova, K. Letters in Organic Chemistry 2006, 3, 176-182.

Kitamura, M.; Suga, S.; Kawai, K.; Noyori, R. Journal of the American Chemical Society 1986, 108, 6071-6072.

12. Kitamura, M.; Okada, S.; Suga, S.; Noyori, R. Journal of the American Chemical Society 1989, 111, 4028-4036.
13. Noyori, R.; Suga, S.; Kawai, K.; Okada, S.; Kitamura, M.; Oguni, N.; Hayashi, M.; Kaneko, T.; Matsuda, Y. Journal of Organometallic Chemistry 1990, 382, 19-37.

14. Kitamura, M.; Suga, S.; Niwa, M.; Noyori, R. Journal of the American Chemical Society 1995, 117, $4832-4842$.

15. Yamakawa, M.; Noyori, R. Journal of the American Chemical Society 1995, 117, 6327-6333.

16. Yamakawa, M.; Noyori, R. Organometallics 1999, 18, 128-133.

17. García Martínez, A.; Teso Vilar, E.; García Fraile, A.; De La Moya Cerero, S.; Martínez Ruiz, P. Tetrahedron: Asymmetry 1998, 9 , 1737-1745.

18. Wu, Z. L.; Wu, H. L.; Wu, P. Y.; Uang, B. J. Tetrahedron: Asymmetry 2009, 20, 1556-1560.

19. Hari, Y.; Aoyama, T. Synthesis 2005, 583-587.

de las Casas Engel, T.; Maroto, B. L.; Martínez, A. G.; de la Moya Cerero, S. Tetrahedron: Asymmetry 2008, 19, $2003-2006$.

Oppolzer, W.; Radinov, R. N. Tetrahedron Letters 1988, 29, 5645-5648.

Sánchez-Carnerero, E. M.; de las Casas Engel, T.; Maroto, B. L.; de la Moya Cerero, S. Tetrahedron: Asymmetry 2009, 20, 26552657.

Martínez, A. G.; Vilar, E. T.; Fraile, A. G.; de la Moya Cerero, S.; Martínez Ruiz, P.; Díaz Morillo, C. Tetrahedron: Asymmetry 2007, 18, 742-749.

24. Stoyanova, M. P.; Shivachev, B. L.; Nikolova, R. P.; Dimitrov, V. Tetrahedron: Asymmetry 2013, $24,1426-1434$.

25.

Mino, T.; Suzuki, A.; Yamashita, M.; Narita, S.; Shirae, Y.; Sakamoto, M.; Fujita, T. Journal of Organometallic Chemistry 2006, 691, 4297-4303.

.

Hui, A.; Zhang, J.; Fan, J.; Wang, Z. Tetrahedron: Asymmetry 2006, 17, 2101-2107.

Hui, A.; Zhang, J.; Sun, H.; Wang, Z. Arkivoc 2008, 2008, 25-32.

Ramón, D. J.; Yus, M. Tetrahedron: Asymmetry 1997, 8, 2479-2496.

Kozakiewicz, A.; Ullrich, M.; Wełniak, M.; Wojtczak, A. Journal of Molecular Catalysis A: Chemical 2010, 326, $128-140$.

Sun, J.; Pan, X.; Dai, Z.; Zhu, C. Tetrahedron: Asymmetry 2008, 19, 2451-2457.

Murtinho, D.; Elisa Silva Serra, M.; Rocha Gonsalves, A. M. d. Tetrahedron: Asymmetry 2010, 21, $62-68$.

Martínez, A. G.; Vilar, E. T.; Fraile, A. G.; De La Moya Cerero, S.; Ruiz, P. M.; Subramanian, L. R. Tetrahedron: Asymmetry 1996, 7, 1257-1260

Hanyu, N.; Aoki, T.; Mino, T.; Sakamoto, M.; Fujita, T. Tetrahedron: Asymmetry 2000, 11, 2971-2979.

Hanyu, N.; Aoki, T.; Mino, T.; Sakamoto, M.; Fujita, T. Tetrahedron: Asymmetry 2000, 11, 4127-4136.

Martínez, A. G.; Vilar, E. T.; Fraile, A. G.; De La Moya Cerero, S.; Maroto, B. L. Tetrahedron: Asymmetry 2003, 14, $1959-1963$. García Martínez, A.; Teso Vilar, E.; García Fraile, A.; De La Moya Cerero, S.; Lora Maroto, B. Tetrahedron: Asymmetry 2004, 15, 753-756.

Chelucci, G.; Soccolini, F. Tetrahedron: Asymmetry 1992, 3, 1235-1238.

Goldfuss, B.; Steigelmann, M.; Khan, S. I.; Houk, K. N. Journal of Organic Chemistry 2000, 65, 77-82.

Xu, Q.; Wang, G.; Pan, X.; Chan, A. S. C. Tetrahedron: Asymmetry 2001, 12, 381-385.

Xu, Q.; Wu, X.; Pan, X.; Chan, A. S. C.; Yang, T. K. Chirality 2002, 14, 28-31.

Goldfuss, B.; Steigelmann, M.; Rominger, F. European Journal of Organic Chemistry 2000, 1785-1792.

Leven, M.; Schlörer, N. E.; Neudörfl, J. M.; Goldfuss, B. Chemistry - A European Journal 2010, 16, 13443-13449.

Genov, M.; Kostova, K.; Dimitrov, V. Tetrahedron: Asymmetry 1997, 8, 1869-1876.

Dimitrov, V.; Simova, S.; Kostova, K. Tetrahedron 1996, 52, 1699-1706.

Dimitrov, V.; Bratovanov, S.; Simova, S.; Kostova, K. Tetrahedron Letters 1994, 35, 6713-6716.

Parrott II, R. W.; Hitchcock, S. R. Tetrahedron: Asymmetry 2008, 19, 19-26.

Kwong, H. L.; Lee, W. S. Tetrahedron: Asymmetry 1999, 10, 3791-3801.

Koning, B.; Buter, J.; Hulst, R.; Stroetinga, R.; Kellogg, R. M. European Journal of Organic Chemistry 2000, $2735-2743$.

Dimitrov, V.; Dobrikov, G.; Genov, M. Tetrahedron: Asymmetry 2001, 12, 1323-1329.

Philipova, I.; Dimitrov, V.; Simova, S. Tetrahedron: Asymmetry 1999, 10, 913-921.

Nugent, W. A. Chemical Communications 1999, 1369-1370.

Knollmüller, M.; Ferencic, M.; Gärtner, P.; Mereiter, K.; Noe, C. R. Tetrahedron: Asymmetry 1998, 9, 4009-4020.

De Oliveira, L. F.; Costa, V. E. U. Tetrahedron: Asymmetry 2004, 15, 2583-2590.

Martins, J. E. D.; Mehlecke, C. M.; Gamba, M.; Costa, V. E. U. Tetrahedron: Asymmetry 2006, 17, 1817-1823.

Martínez, R.; Zoli, L.; Cozzi, P. G.; Ramón, D. J.; Yus, M. Tetrahedron: Asymmetry 2008, 19, 2600-2607.

Dobrikov, G. M.; Philipova, I.; Nikolova, R.; Shivachev, B.; Chimov, A.; Dimitrov, V. Polyhedron 2012, 45, $126-143$.

Kamenova-Nacheva, M.; Dobrikov, G.; Dimitrov, V. Arkivoc 2009, 2009, 141-152.

Salisova, M.; Puciova, M.; Postnova, V. N.; Toma, S. Chemical Papers 1990, 44, 201-207.

Genov, M.; Dimitrov, V.; Ivanova, V. Tetrahedron: Asymmetry 1997, 8, 3703-3706.

Viswanathan, C. T.; Wilkie, C. A. Journal of Organometallic Chemistry 1973, 54, 1-7.

Brandsma, L.; Verkruijsse, H. D. Preparative Polar Organometallic Chemistry; Springer-Verlag: Berlin Heidelberg, 1987 ; Vol. 1.

Thiele, K.-H.; Langguth, E.; Müller, G. E. Zeitschrift für anorganische und allgemeine Chemie 1980, 462, $152-158$. 
64. Dimitrov, V.; Kostova, K.; Genov, M. Tetrahedron Letters 1996, 37, 6787-6790.

65. Koning, B.; Meetsma, A.; Kellogg, R. M. Journal of Organic Chemistry 1998, 63, 5533-5540.

66. Gilman, H.; Gray, S. Journal of Organic Chemistry 1958, 23, 1476-1479.

67. Pinkerton, F. H.; Thames, S. F. Journal of Organometallic Chemistry 1971, 29, C4-C5.

68. Starikova, O. V.; Dolgushin, G. V.; Larina, L. I.; Ushakov, P. E.; Komarova, T. N.; Lopyrev, V. A. Russian Journal of Organic Chemistry 2003, 39, 1467-1470.

69. Dimitrov, V.; Panev, S. Tetrahedron: Asymmetry 2000, 11, 1513-1516.

70. Thiele, K. H.; Krüger, H.; Köhler, E. Zeitschrift für anorganische und allgemeine Chemie 1977, 437, $210-212$.

71. Dobrikov, G. M.; Valcheva, V.; Stoilova-Disheva, M.; Momekov, G.; Tzvetkova, P.; Chimov, A.; Dimitrov, V. European Journal of Medicinal Chemistry 2012, 48, 45-56. 


$$
\text { up to } 96 \% \text { ee }
$$

